\begin{tabular}{|c|l|}
\hline Title & On the dynamics of crystalline motions \\
\hline Author(s) & Giga, Y.; Gurtin, M.E; Matias, J. \\
\hline Citation & Hokkaido University Preprint Series in Mathematics, 336, 1-67 \\
\hline Issue Date & 1996-5-1 \\
\hline DOI & 10.14943/83482 \\
\hline Doc URL & http://hdl.handle.net/2115/69086 \\
\hline Type & bulletin (article) \\
\hline File Information & pre336.pdf \\
\hline
\end{tabular}

Instructions for use 


\section{ON THE DYNAMICS OF CRYSTALLINE MOTIONS}

Y. Giga, M.E. Gurtin and J. Matias

Series \#336. May 1996 


\section{HOKKAIDO UNIVERSITY PREPRINT SERIES IN MATHEMATICS}

\#312 H.M.Ito and T. Mikami, Poissonian asymptotics of a randomly perturbed dynamical system: Flip-flop of the Stochastic Disk Dynamo, 20 pages. 1995.

\#313 T. Nakazi, Slice maps and multipliers of invariant subspaces, 11 pages. 1995.

\#314 T. Mikami, Weak convergence on the first exit time of randomly perturbed dynamical systems with a repulsive equilibrium point, 20 pages. 1995.

\#315 A. Arai, Canonical commutation relations, the Weierstrass Zetafunction, and infinite dimensional Hilbert space representations of the quantum group $U_{q}\left(\mathfrak{s l}_{2}\right), 22$ pages. 1995.

\#316 Y. Shibukawa, Vertex-face correspondence in elliptic solutions of the Yang-Baxter equation, 8 pages. 1995.

\#317 M.-H. Giga and Y. Giga, Consistency in evolutions by crystalline curvature, 16 pages. 1995.

\#318 Wei-Zhi Sun, Shadows of moving surfaces, 19 pages. 1995.

\#319 S. Izumiya and G.T. Kossioris, Bifurcations of shock waves for viscosity solutions of Hamilton-Jacobi equations of one space variable, 39 pages. 1995.

\#320 T. Teruya, Normal intermediate subfactors, 44 pages. 1995.

\#321 M. Ohnuma, Axisymmetric solutions and singular parabolic equations in the theory of viscosity solutions, 26 pages. 1995.

\#322 T. Nakazi, An outer function and several important functions in two variables, 12 pages. 1995.

\#323 N. Kawazumi, An infinitesimal approach to the stable cohomology of the moduli of Riemann surfaces, 22 pages. 1995.

\#324 A. Arai, Factorization of self-adjoint operators by abstract Dirac operators and its application to second quantizations on Boson Fermion Fock spaces, 15 pages. 1995.

\#325 K. Sugano, On strongly separable Frobenius extensions, 11 pages. 1995.

\#326 D. Lehmann and T. Suwa, Residues of holomorphic vector fields on singular varieties, 21 pages. 1995.

\#327 K. Tsutaya, Local regularity of non-resonant nonlinear wave equations, 23 pages. 1996.

\#328 T. Ozawa and Y. Tsutsumi, Space-time estimates for null gauge forms and nonlinear Schrödinger equations, 25 pages. 1996.

\#329 O. Ogurisu, Anticommutativity and spin 1/2 Schrödinger operators with magnetic fields, 12 pages. 1996.

\#330 Y. Kurokawa, Singularities for projections of contour lines of surfaces onto planes, 24 pages. 1996.

\#331 M.-H. Giga and Y. Giga, Evolving graphs by singular weighted curvature, 94 pages. 1996.

\#332 M. Ohnuma and K. Sato, Singular degenerate parabolic equations with applications to the $p$-laplace diffusion equation, 20 pages. 1996.

\#333 T. Nakazi, The spectra of Toeplitz operators with unimodular symbols, 9 pages. 1996.

\#334 B. Khanedani and T. Suwa, First variation of horomorphic forms and some applications, 11 pages. 1996.

\#335 J. Seade and T. Suwa, Residues and topological invariants of singular holomorphic foliations ${ }^{1}$, 28 pages. 1996. 
ON THE DYNAMICS OF CRYSTALLINE MOTIONS

Yoshikazu Giga $¥$, Morton E. Gurtint, and Jose Matias ${ }^{\dagger}$

\author{
tDepartment of Mathematics \\ Hokkaido University \\ Sapporo 060, Japan \\ tDepartment of Mathematics \\ Carnegie Mellon University \\ Pittsburgh, PA 15213, USA \\ †tInstituto Superior Te'cnico \\ Departamento de Matema'tica \\ Avenida Rovisco Pais \\ 1096, Lisboa Codex, Portugal
}

Dedicated to Professor Kôji Kubota on his sixtieth birthday 
ABSTRACT: Solids can exist in polygonal shapes with boundaries unions of flat pieces called facets. Analyzing the growth of such crystalline shapes is an important problem in materials science. In this paper we derive equations that govern the evolution of such shapes; we formulate the corresponding initial-value problem variationally; and we use this formulation to establish a comparison principle for crystalline evolutions. This principle asserts that two evolving crystals one initially inside the other will remain in that configuration for all time.

KEY WORDS: phase transitions, curvature flows, crystalline energies, interfaces, comparison theorems.

RUNNING TITLE: Dynamics of crystalline motions 


\section{INTRODUCTION. \\ a. OVERVIEW}

As is well known, solids can exist in polygonal shapes with boundaries unions of flat pieces called facets. Analyzing the growth of such crystalline shapes is an important problem in materials science. For sufficiently small crystals, surface effects-related to the interface between the crystal and its ancillary phase-dominate bulk effects, a phenomenon that leads one to represent the bulk material through a constant bulk free-energy $U$ for the crystal relative to the other phase. The underlying crystalline structure manifests itself in the dependences of the interfacial free energy $\hat{\gamma}(n)$ and kinetic modulus $\beta(n)$ on the outward unit normal $n$ to the crystal, with $n$ restricted to a finite set consisting of low-energy orientations of the crystal. Taylor [25] proposed an evolution equation for crystalline motion in $\mathbb{R}^{3}$ under the assumptions $U=0, \beta(n)=\hat{\gamma}(n)^{-1}$. Independently, Angenent and Gurtin [2] established a continuum thermomechanical theory of crystal growth; their study, while limited to evolution in $\mathbb{R}^{2}$, does not make the restrictive assumptions regarding $U, \beta(n)$, and $\bar{\gamma}(n), 1$ and leads to an evolution equation consistent with that of Taylor.

The purpose of this paper is threefold: (i) we extend the derivation of [2] to $\mathbb{R}^{3}$; (ii) we formulate the underlying crystal-growth problem variationally in terms of the subdifferential of the total free energy; (iii) we use this formulation to establish a comparison principle for crystalline motions which, roughly speaking, asserts that two evolving crystals one initially inside the other will remain in that configuration for all time.

\section{b. BASIC EQUATIONS}

We begin by deriving an evolution equation for crystalline surfaces. ${ }^{2}$ Let $\&$ be a crystalline motion with duration T; i.e., $\&$ is a smooth oneparameter family $\delta(t), 0 \leq t<T$, of crystalline surfaces

$$
\delta(t)=\bigcup_{a=1}^{A} F_{a}(t)
$$

\footnotetext{
${ }^{1}$ Cf. $[29]$.

${ }^{2}$ For convenience, throughout the introduction we restrict attention to bounded crystals.
} 
( $t=$ time) such that each (closed) facet $F_{a}(t)$ has unit outward normal (orientation) $\mathrm{n}_{\mathrm{a}}$ independent of $t$. We restrict attention to crystalline motions whose orientations are confined to a finite set $\eta \subset S^{2}$ of admissible orientations; $\boldsymbol{\eta}$ is related to the lattice structure of the crystal and should be envisaged as representing stable orientations of the interface.

Using continuum thermomechanical arguments in conjunction with suitable constitutive assumptions we derive a basic balance law for crystalline motions,

$$
\operatorname{div}_{B} c=U+\beta(n, V) V
$$

in which $V(x, t)$ is the normal velocity of $\delta(t)$ in the direction of its orientation $\mathrm{n}(\mathrm{x}, \mathrm{t})$; $\mathrm{U}$ represents the bulk free-energy of the crystal; $c(x, t) ; a$ tangential vector field, is a surface shear that enters the theory through the capillary stress ${ }^{3}$

$$
C=\hat{\gamma}(n) P+n \otimes c \quad(P=1-n \otimes n)
$$

with $\hat{\gamma}(n)$ the interfacial free energy (here equal to the surface tension); and $\beta(n, V)$ is a kinetic modulus. The balance (1.2) is required to hold away from the edges of the crystal; force balance across the edges of the crystal yields the edge condition

$$
c_{a} \cdot v_{a}=c_{a b}
$$

on each edge $(a, b)$ of any given facet $F_{a}$. Here $b$ labels the facets $F_{b}$ adjacent to $F_{a}, v_{a}$ is the outward unit normal to $\partial F_{a}$ (in the plane of $F_{a}$ ), and the $c_{a b}$ are constants defined by

$$
c_{a b}=\operatorname{sgn}\left(\nu_{a} \cdot n_{b}\right)\left[\left(n_{a} \cdot n_{b}\right) \hat{\gamma}\left(n_{a}\right)-\dot{\gamma}\left(n_{b}\right)\right] /\left[1-\left(n_{a} \cdot n_{b}\right)^{2}\right]^{\frac{1}{2}}
$$

with $\mathrm{n}_{\mathrm{a}}$ the orientation of $\mathrm{F}_{\mathrm{a}}$. The derivation of (1.2)-(1.5) parallels the corresponding derivation of [2] for crystalline curves in the plane. We ${ }^{3}$ We use the term vector for element of $\mathbb{R}^{3}$ and the term tensor for linear transformation of $\mathbb{R}^{3}$ into $\mathbb{R}^{3}$. We write 1 for the identity tensor, $C^{\top}$ for the transpose of a tensor $C$, and $a \otimes b$ for the tensor product of vectors $a$ and $b$. 
assume throughout that

$$
\hat{\gamma}(n)>0, \quad \beta(n, V)>0 \text { is independent of } V, \quad \beta(n)>0 .
$$

If we integrate (1.2) over each facet and use (1.4), we arrive at an evolution equation

$$
\beta_{a} V_{a}(t)=\Lambda_{a}(t)-U
$$

for each facet $F_{a}$, where

$$
\Lambda_{a}(t)=A_{a}(t)^{-1} \sum_{b} c_{a b} L_{a b}(t)
$$

with $L_{a b}$ the length of the $(a, b)$ edge, $A_{a}$ the area of $F_{a}, V_{a}$ the normal velocity of $F_{a}$, and $\beta_{a}=\beta\left(n_{a}\right)$. The quantity $\Lambda_{a}(t)$, called the (energetically-) weighted curvature of $F_{a}$, appears first in [25] (cf. [26], [29]).

\section{c. LOCAL EXISTENCE}

We next analyze crystalline motions governed by the evolution equation (1.7). Here we consider as prescribed: a set $\mathfrak{n}$ of admissible orientations; for each $n \in \mathbb{N}$, an interfacial free energy $\hat{\gamma}(n)>0$ and a kinetic modulus $\beta(n)>0$; and a (constant) bulk free energy $U$. In contrast to the planar case, crystalline motions governed by (1.7) have not been analyzed in the past. Here the first question that arises concerns the solvability of (1.7) for given initial data $8(0)$ with orientations in $\pi$. The equations (1.7) are equivalent to a system of ODEs for the position vectors $x_{i}(t), i=1, \ldots, C$, of the corners of $s(t)$. However, in contrast to the evolution of crystalline curves, this system is generally overdetermined and cannot be solved even. locally in time. (Cf. the counterexample given in Theorem 6.2; we believe that actual crystalline motions would overcome this difficulty through the emergence of new facets.) On the other hand, we establish local existence for (admissible) crystalline motions consistent with (1.7) provided each corner on the initial crystal $8(0)$ is a triple junction (Theorem 6.1), in which case the system of ODEs is well posed. 


\section{d. MAXIMUM PRINCIPLE. COMPARISON}

A-major goal of ours is to establish a comparison principle for crystalline motions governed by (1.7). Such a principle has been derived for the evolution of crystalline curves in the plane [16]; as there, the key step is to establish, for crystalline surfaces, a maximum principle which, modulo suitable hypotheses, has the form:

Maximum Principle. Let $\delta_{1}$ and $\delta_{2}$ be crystalline surfaces such that $\delta_{1}$ touches $\delta_{2}$ at $x_{0}$ from inside. Then:

(i) (a) There are facets $F_{1}$ and $F_{2}$ of $S_{1}$ and $\delta_{2}$ such that $x_{0} \in F_{1} \cap F_{2}$ and such that the orientation of $F_{1}$ equals that of $F_{2}$. (i)(b) The set of orientations of facets of $\delta_{1}$ meeting at $x_{0}$ equals the corresponding set for $\mathrm{S}_{2}$.

(ii) If $F_{1}$ and $F_{2}$ are facets of $s_{1}$ and $s_{2}$ with $x_{0} \in F_{1} \cap F_{2}$ and with the orientation of $F_{1}$ equal to that of $F_{2}$, then the weighted curvatures $\Lambda_{1}$ and $\Lambda_{2}$ of $F_{1}$ and $F_{2}$ satisfy $\Lambda_{1} \leq \Lambda_{2}$.

For smooth surfaces (i) corresponds to the comparison of first derivatives of functions whose graphs coincide with the surfaces $\&_{i}$, (ii) corresponds to the comparison of second derivatives.

Fundamental to a precise statement of this principle is an appropriate class of crystalline surfaces. We define such a class with the aid of hypotheses concerning the edges and corners of the crystals under consideration, hypotheses that render the crystal compatible with the interfacial energy $\hat{\gamma}(\mathrm{n})$.

An important concept in the discussion of interfacial energies is the Frank diagram $\mathcal{F}$, which is the boundary of the convex hull of the set $g=\left\{\hat{\gamma}(m)^{-1} m: m \in \mathbb{N}\right\}$. F is a polygonal region whose vertices belong to $g$. In fact, we assume that $\delta=\{$ vertices of $\mathcal{F}\}$, as then $\pi$ coincides with the set of orientations of the corresponding Wulff crystal. We say that a crystalline surface $\&$ is admissible if the orientations of adjacent facets correspond to adjacent vertices of $\mathcal{F}$, strongly admissible if, in addition, given any corner $c$ of $\delta$, the set of orientations of facets that meet at $c$ correspond to the complete set of vertices of a face of $\mathcal{F}$.

For strongly admissible crystals we are able to prove (i)(a) of the maximum principle as well as a modified version of (i)(b) (Section 10): 
(i)(c) There is a point $y_{0}$ sufficiently close to (and possibly equal to) $x_{0}$ such that $\delta_{1}$ touches $\delta_{2}$ at $y_{0}$ from inside and such that the set of orientations of facets of $\delta_{1}$. meeting at $y_{0}$ equals the corresponding set for $8_{2}$.

Our method of proving $(i)(a)$ and $(i)(c)$ is to exhaust all possible touchings of $\delta_{2}$ by $\delta_{1}$ from inside. For example, strong admissibility excludes the possibility that a corner of $\delta_{1}$ touch the interior of a facet of $\delta_{2}$.

The proof of (ii) is far more difficult. In contrast to crystalline evolution in the plane [16], the complicated nature of the weighted curvature (1.8) makes a direct comparison of surfaces difficult, and for that reason we give a subdifferential characterization of the weighted curvature.

The total free-energy for a crystalline surface $\&$ enclosing a region $\Gamma$ is given by

$$
\mathrm{Uvol} \Gamma+\int_{\delta} \hat{\gamma}(n)
$$

Suppose that $\&$ is represented as the graph of a function $u$ near a facet $F_{a}$. Then that portion of the energy associated with $u$ is represented by the integral

$$
\Phi(u)=\int_{\Omega}[j(\nabla u)+U u]
$$

where $j(q)=\gamma_{0}(-q, 1), \gamma_{0}$ is a natural extension of $\hat{\gamma}$ to $\mathbb{R}^{3}$ (Section $5 b$ ), and $\Omega$ is a neighborhood of the projection $P\left(F_{a}\right)$ of $F_{a}$ on the plane on which $u$ is defined. We consider $\Phi$ on the Hilbert space $H=L^{2}(\Omega)$ corresponding to null Dirichlet boundary data; this definition and a modification of $j$ for large $q$ allows us to consider $\Phi$ as a lower semicontinuous convex function on $\mathrm{H}$. This modification is inconsequential, as the functions $u$ that correspond to crystalline surfaces are Lipschitz continuous.

Next we show that for $\&$ admissible there is a unique $f \in \partial \Phi(u)$ such that 


$$
\|f\|=\inf \{\|h\|: h \in \partial \Phi(u)\}, \quad(\|\cdot\|=\operatorname{norm} \text { on } H) .
$$

We write $f=\partial^{\circ} \Phi(u)$ and refer to $\partial^{\circ} \Phi(u)$ as the canonical restriction of $\partial \Phi(u)$ at $u$. A key step in our argument is to characterize the weighted curvature in terms of the canonical restriction:

$$
\partial^{\circ} \Phi(u)=-\Lambda_{a}+U
$$

on $\Omega_{a}=P\left(F_{a}\right)$ (Theorem 9.1). Such a formula is derived in [13] for $u$ a function of a single spatial variable. (Note that, by $(1.12), \partial^{\circ} \Phi(u)$ is independent of the choice of coordinate system used to describe $\&$ as a graph.)

The central steps in the derivation of (1.12) is to first note that the characterization (1.11) of $\partial^{\circ} \Phi(u)$ implies that

$$
\partial^{\circ} \Phi(u)=-\operatorname{div} \eta+U, \quad \eta(\mathbf{x}) \in \partial j(\nabla u(\mathbf{x}))
$$

for some $\eta$. With this in mind, we construct a piecewise linear function $\eta$ such that, on $\Omega_{a}$, $\operatorname{div} \eta=\Lambda_{a}$ and $\eta(x) \in \partial j(\nabla u(x))$, and then show that, for this $\eta,-\operatorname{div} \eta+U$ minimizes $\|h\|$ for $h \in \partial \Phi(u)$.

A corollary of the existence of $\eta$ is the existence of a (not necessarily continuous) piecewise linear vector field $\boldsymbol{E}$ (the Cahn-Hoffman vector) on \& such that: (j) $\xi \in \partial \gamma(n)$ on each facet $F_{a}$ of $8 ;$ (ii) $\operatorname{div} P \xi=-\Lambda_{a}$ on each facet $F_{a}$, where $P$ is here the projection onto the plane containing $F_{a}$; (iii) for each edge $l$, the projection of $\xi$ onto the plane perpendicular to $l$ is continuous across $l$ (for $\&$ admissible).

We use (1.12) to compare weighted curvatures of touching facets. Indeed, applying the general theory of nonlinear semigroups [5] to general order preserving parabolic systems yields the conclusion that

$$
-\partial^{\circ} \Phi_{1}\left(u_{1}\right) \leq-\partial^{\circ} \Phi_{2}\left(u_{2}\right) \quad \text { in } \Omega^{\prime}
$$

if $u_{1} \leq u_{2}$ in $\Omega$ and $u_{1}=u_{2}$ in an open set $\Omega^{\prime} \subset \Omega$; otherwise $u_{1}<u_{2}$ (Lemma 10.2). This together with (1.12) yields a curvature comparison if $\delta_{1}$ touches $\delta_{2}$ from inside over $F_{1} \cap F_{2} \quad\left(F_{i}\right.$ a facet of $\left.\delta_{i}\right)$ with $F_{1} \cap F_{2}$ a nontrivial polygon. The general case encountered in (ii) can be reduced to this case by strong admissibility. This is a rough sketch of the proof of (ii) 
provided $\delta_{1}$ and $\delta_{2}$ may be represented as graphs near touching facets, edges, or corners. A simple sufficient condition that ensures such representations is that any pair of adjacent orientations in $\eta$ be acute, or, more succintly, that $\eta$ be acute.

Our maximum principle may therefore be stated precisely as follows:

Theorem. Let $\delta_{1}$ and $\delta_{2}$ be strongly admissible crystalline surfaces such that $\&_{1}$ touches $\&_{2}$ at $\mathrm{x}_{0}$ from inside. Then $(i)(a)$ and (i)(c) are satisfied. If, in addition, $\boldsymbol{n}$ is acute, then (ii) is satisfied.

This result is stated as Theorem 10.2. Granted the maximum principle, it is not difficult to establish the comparison

Theorem. Assume that the set $\pi$ of admissible orientations is acute. Let $\delta_{1}$ and $\delta_{2}$ be strongly admissible crystalline motions with common duration $\mathrm{T}$ and with all corners triple junctions. Assume that $s_{2}(0)$ encloses $s_{1}(0)$. Then $s_{2}(t)$ encloses $s_{1}(t)$ for $0 \leq t<T$.

A more general version of this theorem is stated as Theorem 10.1 . The proof of Theorem 10.1 closely parallels the proof of comparison given in [16].

For the existence of strongly admissible crystalline surfaces with all corners triple junctions we need to restrict the shape of the Frank diagram F. Since the set of orientations of facets that meet at a corner corresponds to the complete set of vertices of a face of $\mathcal{F}$, the faces of $\mathcal{F}$ should be triangles. There is a large class of crystalline energies whose faces are triangles and for which $\pi$ is acute.

As Cahn and Taylor (cf. [28]) have pointed out, there is an admissible crystalline surface $\&$ of minimal surface free energy that is not strongly admissible; more precisely, $\&$ is not strongly admissible near a corner $c$, and its surface energy cannot be decreased by modifying the surface near c by inserting facets, edges or corners. In our work strong admissibility is used only to classify possible touchings. (For example, we do not use strong admissibility to establish the existence of the Cahn-Hoffman vector field.)

Crystalline motion in the plane, as governed by the planar version of (1.7), has been studied. As noted in [2], the evolution equation then reduces to a system of ordinary differential equations for admissible crystalline mo- 
tions, and local existence of solutions is not difficult, although one or more facets may disappear in finite time $\tau$. However, at such a $\tau$, the shape is admissible (cf., e.g., [18]), and one can extend the solution beyond $\tau$. Such an extended solution is termed weakly admissible by [16], who establish a comparison principle for such evolutions. Applying this result, large time behavior is studied in [16] for the case in which the sign of $U$ is such that a sufficiently large crystal grows. The asymptotic shape of the crystal is then the Wulff shape corresponding to $1 / \beta$, a result proved for a smooth energy $\gamma$ in [23]. When $U=0$, a bounded convex polygon shrinks. It is shown in [24] that this shrinking is asymptotically similar to the shrinking of the Wulff shape of $\gamma$ when $\beta=\gamma^{-1}$, provided the initial polygon has at least five corners.

Crystalline motions are useful in computing evolutions of curves by smooth energy $\gamma$, by approximating the Frank diagram $\mathcal{F}$ of $\gamma$ by a polygon. The crystalline motion then approximates the original motion, at least when the initial curve is the graph of a function of one variable, and provided $U=0$ (cf. [18], [19], [13]; [18] obtains the convergence rate), and the analysis extends to closed convex curves (cf. [17]). For more details and a discussion of the background of the problem, see the recent review by Girão and Kohn [19] and the references therein.

It is interesting to study the evolution when the initial data is not admissible; that is, when this data has a curved portion. A general definition of solutions may be given via nonlinear semigroup theory when $U=0$ and when the initial curve is the graph of a periodic function (cf. [13]); using this, a unique global solution is constructed in [13] for general nonadmissible initial data, and the speed of facets agrees with (1.7). Recently, [11] have shown that weakly admissible crystalline motions are actually solutions as defined in [13]. Unfortunately, the method in [13] does not apply to the case $U \neq 0$. Reciently, a new definition of solutions, reflecting the theory of viscosity solutions, is given in [14] for the initial curve the graph of a function. As announced there, a unique global solution may be constructed for general nonadmissible (periodic) initial data even if $U \neq 0$. In that case (cf. [15]) our weakly admissible solution is a solution as defined in [14]. These results suggest that it is natural to assume that a facet remains a facet during its evolution, at least for constant $U$.

In [3] a semi-discretized implicit scheme is proposed to construct 
solutions of the planar version of (1.7) when the crystal is not restricted as a graph. The time is discretized, and at each time-step the value of a solution is determined via a variational problem. This scheme does not require that a facet remain a facet. Moreover, the scheme is consistent with that for the case of smooth energy $\gamma$, where convergence to a generalized solution is proved (cf. [4]). It is shown in [3] that the approximate solution converges to the solution of (1.7) if that solution is admissible; this gives a further justification of the notion of admissibility. 


\section{CRYSTALLINE MOTIONS}

By a crystal we mean a possibly unbounded polyhedron $\Gamma$ in $\mathbb{R}^{3}$; its boundary $\delta=\partial \Gamma$ is called a crystalline surface and the outward unit normal $n$ to $\Gamma$ is called the orientation of 8 . By definition, 8 is the union

$$
\delta=\bigcup_{a=1}^{A} F_{a}
$$

of a finite number of closed (maximally) connected flat sides $F_{a}$ called facets; adjacent facets intersect along closed line segments called edges, and edges intersect in corners. If $F_{a}$ and $F_{b}$ are adjacent facets, then we refer to the corresponding edge as the $(a, b)$-edge. Given a field $\varphi$ on $\delta$, we write $\varphi_{q}, q=a, b$, for the limit of $\varphi$ as the (a,b)-edge is approached from the facet $F_{\mathrm{q}}$ :

$$
\varphi_{\mathrm{q}}(\mathbf{x})=\lim _{\substack{y \rightarrow \mathbf{x} \\ \mathbf{y} \in \mathrm{F}_{\mathrm{q}}}} \varphi(y)
$$

To the definition of a crystal $\Gamma$ we add the requirement that if $\Gamma$ is unbounded, then each unbounded facet $F$ of $\Gamma$ is regularly unbounded in the following sense: $F$ has at most two unbounded edges, and if $F$ has two unbounded edges, then the unbounded edges are not parallel. Then, if $F^{\varepsilon}$ denotes the intersection of an unbounded facet $F$ with a ball of radius $\varepsilon$,

$$
\text { perimeter }\left(F^{\varepsilon}\right) / \text { area }\left(F^{\varepsilon}\right) \rightarrow 0 \text { as } \varepsilon \rightarrow 0 \text {. }
$$

We add this restriction for convenience as it simplifies the discussion; it rules out unbounded strip-like facets for which (2.3) has a nonzero limit.

Let $V$ be a vector field and $C$ a tensor field, with both fields defined on 8 . Then the surface divergence $\operatorname{div}_{\delta} v$ is defined on each facet of 8 in the standard manner, while $\operatorname{div}_{8} \mathrm{C}$ is defined on each facet by the relation

$$
a \cdot \operatorname{div}_{8} C=\operatorname{div}_{8}\left(C^{\top} a\right)
$$


for all vectors a.

We write

$$
P=1-n \otimes n
$$

$P(x)$ is the projection onto the tangent plane for $\&$ at $\mathbf{x}$.

A crystalline motion \& (with duration $T$ ) is a smooth oneparameter family $\&(t), 0 \leq t<T$, of crystalline surfaces

$$
\delta(t)=\bigcup_{a=1}^{A} F_{a}(t)
$$

such that each facet $F_{a}(t)$ has orientation $n_{a}$ independent of $t$, and such that the position vector of each corner varies smoothly in time. In such motions facets are neither created nor destroyed. We will generally consider $s(t)$ as an interface between the enclosed crystal $\Gamma(t)$ and the region exterior to $\Gamma(t)$, which represents the ancillary phase. We denote by $V(x, t)$ the normal velocity to $s(t)$ in the direction of its orientation $n(x, t)$; since the facet normals are constant, each facet $F_{a}(t)$ has normal velocity $V_{a}(t)$ a function only of $t$. Finally, we write

$$
s_{T}=\{(\mathbf{x}, t): \mathbf{x} \in \delta(t), \quad t \in(0, T)\},
$$

and given a scalar field $\varphi$ on $\delta_{\mathrm{T}}$, we denote by $\varphi^{\circ}$ the normal time derivative of $\varphi$ (the derivative following the normal trajectories of $\delta(t)$ ):

Let $b$ be an evolving subsurface of 8 ; that is, $b(t) \subset s(t)$ for all $t$ in an open time-interval with $\partial \mathcal{l}(t)$ a bounded, continuous, piecewisesmooth curve that admits local parametrizations $x=r(\lambda, t)$ that are continuous, piecewise-smooth functions of $(\lambda, t)$. Then $\partial \ell(t)$ has a well defined outward unit normal $v(x, t)$ that is tangential to $8(t)$, and, given a local parametrization, the fjeld $w(x, t)=\partial r(\lambda, t) / \partial t$ satisfies

$$
w \cdot n=V, \quad w \cdot v=w_{\partial l}(\tan ),
$$

where $W$ ol(tan), the tangential edge velocity of $b(t)$, is independent of 
the choice of parametrization. The motion of $\partial b(t)$ may be characterized intrinsically by the velocity field

$$
w_{\partial \ell}=V_{n}+w_{\partial \ell(\tan )^{\nu}} .
$$

More generally, we will use the term admissible velocity field for de to denote vector fields $w(x, t)$ consistent with $(2.7)$ on dh(t). Similarly, we may define the tangential edge velocity $W_{\partial F}(\tan )$ and intrinsic velocity $w_{\partial F}$ for any facet $F(t)$; then, for $F_{a}(t)$ and $F_{b}(t)$ adjacent facets,

$$
w_{\partial F_{a}}=w_{\partial F_{b}}
$$

on the $(a, b)$ - edge.

Given a facet $F$, let $\varphi(x, t)$ be smooth in $(x, t)$ for $x \in F(t)$. Then the following transport identity is valid for evolving subsurfaces $b$ of $\&$ with $b(t) \subset F(t)$ :

$$
(d / d t) \int_{\ell(t)} \varphi=\int_{\ell(t)} \varphi^{0}+\int_{\partial \ell(t)} \varphi w_{\partial \ell(\tan )} .
$$

Finally, we have the standard identity, valid whenever the crystal $\Gamma(t)$ is bounded:

$$
(d / d t) \operatorname{vol} \Gamma(t)=\int_{\delta(t)} V
$$


3. FORCES. CAPILLARY STRESS. CAHN-HOFFMAN VECTOR

Let \& be a crystalline motion with orientation $n$ and duration $T$. We associate with \& a tensor field $\mathrm{C}(\mathrm{x}, t)$, the capillary stress, and a vector field $\pi(x, t)$, the internal force. These fields, defined for all $(x, t) \in \delta_{T}$, are required to satisfy the force balance

$$
\int_{\partial b} C \nu+\int_{l} \pi=0
$$

for all evolving subsurfaces $b$ of $\&$, where $v$ is the outward unit normal to $\partial l$. The first integral gives the force on $l(t)$ exerted across $\partial \ell(t)$ by the portion of $\delta(t)$ exterior to $\ell(t)$; the second integral gives the force exerted on $b(t)$ by the bulk material adjacent to the interface. Although $\mathrm{Cv}$ is defined on each vector $v$, its action on vectors normal to $s(t)$ is irrelevant, and for that reason we add the restriction

$$
c_{n}=0
$$

The requirement ( 3.1 ) is equivalent to the local balance

$$
\operatorname{div}_{8} C+\pi=0
$$

on each facet ${ }^{4}$ in conjunction with the edge balance

$$
C_{a} \nu_{a}=-C_{b} \nu_{b}
$$

across each edge (a,b), where we have used the notation (2.2), and where, for $q=a, b, \nu_{q}$ is the outward unit normal to the boundary curve $\partial F_{q}$.

Given any evolving subsurface $b$ of 8 , the rate at which the capillary stress does work on $b$ is given by

$$
\int_{\partial l}^{C} \boldsymbol{C} \cdot \mathrm{w},
$$

where $w$ is an admissible velocity field for $\partial$. This term will enter our ${ }^{4}$ The assertion that a differential equation hold on a facet is meant to signify that the equation hold on the interior of the facet. 
statement of the second law, and represents the only term in that law involving de. For that reason we assume that (3.5) is independent of the choice of admissible velocity field $w$, and hence of the choice of parametrization for $\partial \mathcal{l}(t)$. Then, by (iii) of the Invariance Lemma of Gurtin and Struthers [21], $C$ may be written in the form

$$
C=\sigma P+n \otimes c
$$

with $\sigma$ a scalar field and $c$ a tangential vector field. The action $C \nu$ of $C$ on a tangential field $v$ then consists of a component $\sigma v$ tangent to the surface and a component $(c \cdot v) n$ normal to the surface, so that $\sigma$ is the surface tension, while $c$ represents the surface shear.

Choosing the intrinsic velocity (2.8) for $w$, we may rewrite $(3.5)$ as

$$
\int_{\partial l} C v \cdot w_{\partial l}=\int_{\partial l}\left\{\sigma w_{\partial l(\tan )}+V c \cdot v\right\}
$$

Further, if $\ell(t)$ is contained in the union of adjacent facets $F_{a}(t)$ and $F_{b}(t)$, then, writing $b_{a}(t)=b(t) \cap F_{a}(t)$ and similarly for $b_{b}(t)$, we may use (2.9) and (3.4) to conclude that

$$
\int_{\partial l} C v \cdot w_{\partial l}=\int_{\partial b_{a}} C v \cdot w_{\partial l_{a}}+\int_{\partial l_{b}} C v \cdot w_{\partial l_{b}} \cdot
$$

Since $\mathbf{n}$ is constant on each facet,

$$
n \cdot \operatorname{div}_{8} C=\operatorname{div}_{8} c ;
$$

thus, writing

$$
\pi=\pi \cdot n,
$$

the normal component of the force balance (3.3) has the form

$$
\operatorname{div}_{8} c+\pi=0
$$


on each facet. As we shall see, the surface tension $\sigma$ will be a prescribed constant on each facet (cf. (4.3) and (5.3)); granted this the tangential component of (3.3) is satisfied identically on each facet provided

$$
P \pi=0 \text {. }
$$

We henceforth assume that (3.12) is satisfied.

By (3.6), the balance (3.4) is equivalent to the relation

$$
\dot{\sigma}_{a} \nu_{a}+\left(c_{a} \cdot \nu_{a}\right) n_{a}=-\left[\sigma_{b} \nu_{b}+\left(c_{b} \cdot \nu_{b}\right) n_{b}\right]
$$

which may be solved (uniquely) for $c_{a} \cdot v_{a}$ and $c_{b} \cdot v_{b}$ : indeed, since $\nu_{a} \cdot n_{b}=v_{b} \cdot n_{a}$, taking the inner product of (3.13) with $n_{a}$ and $n_{b}$ and solving the resulting equations for $c_{a} \cdot v_{a}$ yields

$$
\left[1-\left(n_{a} \cdot n_{b}\right)^{2}\right] c_{a} \cdot v_{a}=\left[\left(n_{a} \cdot n_{b}\right) \sigma_{a}-\sigma_{b}\right] \nu_{a} \cdot n_{b}
$$

hence

$$
c_{a} \cdot v_{a}=\operatorname{sgn}\left(v_{a} \cdot n_{b}\right)\left[\left(n_{a} \cdot n_{b}\right) \sigma_{a}-\sigma_{b}\right] /\left[1-\left(n_{a} \cdot n_{b}\right)^{2}\right]^{\frac{1}{2}} .
$$

In view of (3.6), C is characterized by the vector field

$$
\xi=\sigma n-c
$$

which we refer to as the Cahn-Hoffman vector for $C$; in fact, $\sigma=\boldsymbol{\xi} \cdot \mathbf{n}$ and $c=-P E$, so that

$$
C=(\xi \cdot n) P-n \otimes P \xi
$$

Thus, by (3.11),

$$
-\operatorname{div}_{S}(P \xi)+\pi=0, \quad \xi \cdot n=\sigma
$$

on each facet. Further, substituting (3.17) into (3.4), and then acting on the resulting equation with the rotation that carries $\nu_{a}$ into $n_{a}$ and $n_{a}$ into $-v_{a}$, we are led to 


$$
\left(\xi_{a} \cdot n_{a}\right) n_{a}+\left(\xi_{a} \cdot \nu_{a}\right) \nu_{a}=\left(\xi_{b} \cdot n_{b}\right) n_{b}+\left(\xi_{b} \cdot \nu_{b}\right) \nu_{b}
$$

hence the projection of $\boldsymbol{E}$ onto the plane perpendicular to the $(\mathrm{a}, \mathrm{b})$-edge is continuous across the (a,b)-edge. If $\xi$ satisfies (3.18) on each facet and (3.19) on each edge, then $c=-P \xi$ satisfies (3.11) on each facet and (3.15) on each edge, and vice versa. 


\section{ENERGETICS}

Let $\&$ be a crystalline motion with orientation $\mathrm{n}$ and duration $\mathrm{T}$. We associate with $\&$ an interfacial free energy $\gamma(x, t)$, per unit area, defined for each $(x, t) \in \delta_{T}$, and a constant $U$ that represents the bulk free energy of the crystal relative to that of the ancillary phase. Within the purely mechanical framework considered here the second law is the assertion that, given any evolving subsurface $l$ of $8-$ if we consider $b$ as an infinitesimally thin region whose "boundary" consists of surfaces $b+0$ n and $b-0 n$ that lie in the bulk material - then the rate at which the free energy of $b$ is changing minus the rate at which bulk energy is lost by $l$ across its "boundary" is not greater than the rate at which forces do work on $\ell$; precisely,

$$
(d / d t)\left\{\int_{\ell(t)} \gamma\right\}+\underset{\ell(t)}{U} \mathrm{C} V \leq \int_{\partial \ell(t)} C \nu \cdot w \partial \ell
$$

where $v$ is the outward unit normal to $\partial l$. The force $\pi$ does not appear in (4.1), as its action is internal to the "region" $l$.

Suppose that $b(t)$ is contained in a facet $F(t)$. Then using (2.10), (2.11), (3.7), and (3.10) we may rewrite (4.1) as

$$
\int_{\ell(t)}\left[\gamma^{\circ}+(\pi+U) V\right] \leq \int_{\partial \ell(t)}(\sigma-\gamma) w_{\partial \ell(\tan )} .
$$

Given any $t_{0}$, any sufficiently regular subset $b_{0}$ of $F\left(t_{0}\right)$, and any smooth scalar field $\varphi$ on $\partial b_{0}$, we can always find an evolving subsurface $b$ contained in $F$ such that $b\left(t_{0}\right)=l_{0}$ and $w_{\partial \ell(\tan )}\left(x, t_{0}\right)=\varphi(x)$ on $\partial b_{0}$. We are therefore led to two important conclusions: the surface tension and interfacial free energy coincide,

$$
\sigma=\gamma
$$

the dissipation inequality

$$
\gamma^{\circ}+(\pi+U) V \leq 0
$$


hold on each facet.

Conversely, if (4.3) and (4.4) are satisfied, then (4.2) and hence (4.1) hold for each evolving subsurface that is contained in a single facet, and we may use (3.8) to show that (4.1) holds for all evolving subsurfaces.

Finally, we note that, for a bounded crystal, (4.1) applied with $\ell(t)=s(t)$ and $(2.11)$ yield

$$
(d / d t)\left\{U \text { vol } \Gamma(t)+\int_{\delta(t)} \gamma\right\} \leq 0
$$

so that the total free energy decreases with time. 
5. CONSTITUTIVE EQUATIONS. FRANK DIAGRAM. CONVEXIFIED ENERGY

a. THERMODYNAMICALLY RESTRICTED CONSTITUTIVE EQUATIONS

We now restrict attention to crystalline surfaces and motions whose orientations are confined to a finite set $n \subset S^{2}$ of admissible orientations. The set $\pi$ is related to the lattice structure of the crystal and should be envisaged as representing stable orientations of the interface. As constitutive equations we allow the free energy $\gamma$ and the normal accretive force $\pi$ to depend on the orientation and kinetics of the interface through dependences on $n$ and $V$ :

$$
\gamma=\hat{\gamma}(n, V), \quad \pi=\hat{\pi}(n, V)
$$

i.e., e.g., $\gamma(x, t)=\hat{\gamma}(n(x, t), V(x, t))$. We assume that $\hat{\gamma}$ and $\hat{\pi}$, with domain $\Omega \times \mathbb{R}$, are smooth functions of $V$.

We require that (5.1) be consistent with the dissipation inequality (4.4). Then for $F(t)$ a facet with orientation $n \in \mathbb{R}$,

$$
\hat{\gamma}_{V}(n, V) V^{0}+[\hat{\pi}(n, V)+U] V \leq 0
$$

an inequality that holds for all such facets if and only if:

(i) $\hat{\gamma}(n, V)$ is independent of $V$,

$$
\gamma=\hat{\gamma}(n)
$$

(ii) there is a kinetic modulus $\beta(n, V) \geq 0$ such that

$$
\hat{\pi}(n, V)=-U-\beta(n, V) V
$$

We assume, henceforth, that (5.3) and (5.4) are satisfied with

$$
\hat{\gamma}(n)>0, \beta(n, V) \text { independent of } V, \beta(n)>0 \text {. }
$$

b. FRANK DIAGRAM. CONVEXIFIED ENERGY

The Frank diagram $F$, which is the boundary of the convex hull of 
the finite set

$$
g=\left\{\hat{\gamma}(n)^{-1} n: n \in \mathbb{R}\right\}
$$

is a polyhedral surface whose vertices belong to $g$. We will, in fact, assume throughout that:

$$
g \text { is the set of vertices of } \mathcal{F}
$$

(cf. (5.12)). The construction of $\mathcal{F}$ ensures that each $n \in S^{2}$ correspond to a unique point Frank $(n)=\alpha(n) n \in \mathcal{F}, \alpha(n)>0$. Thus and by (5.7), the strictly positive function $\hat{\gamma}_{0}$ on $S^{2}$ defined by

$$
\operatorname{Frank}(n)=\hat{\gamma}_{0}(n)^{-1} n
$$

extends $\hat{\gamma}$ from $\eta$ to $S^{2}$, and $\mathcal{F}=\left\{\hat{\gamma}_{0}(n)^{-1} n: n \in S^{2}\right\}$. It is convenient to further extend $\hat{\gamma}$ to $\mathbb{R}^{3}$ by homogeniety: $\gamma_{0}(0)=0$ and

$$
\gamma_{0}(p)=|p| \hat{\gamma}_{0}(p /|p|)
$$

for all $p \in \mathbb{R}^{3}, p=0$. Then

$$
\mathcal{F} \text { is the one-level set of } \gamma_{0} \text {, }
$$

so that $\gamma_{0}$ is a convex function. We will refer to $\gamma_{0}$ as the convexified energy.

Both the Frank diagram and the crystal are polyhedral; to avoid confusion we will use the following differences in terminology:

$\begin{array}{ll}\text { crystal } & \text { Frank diagram } \\ \text { facet } & \text { face } \\ \text { corner } & \text { vertex }\end{array}$

We will refer to $\pi \subset \pi$ as compatible if Frank( $M)$ is contained in a face $f$ of $\mathcal{F}$; by (5.6) and (5.7). Frank $(n)$ is then a vertex of $f$ for each $n \in \mathbb{M}$; if Frank(M) is the set of all vertices of $f$, then $\mathbb{M}$ is complete. 
Finally, $m, n \in \mathcal{N}$ are adjacent if the line segment from Frank(m) to Frank $(n)$ is an edge of $\mathcal{F}$ (in which case $\{m, n$ ) is compatible); and we will refer to $\boldsymbol{n}$ as acute if $m \cdot n>0$ for every pair of adjacent orientations $\boldsymbol{m}, \boldsymbol{n} \in \boldsymbol{N}$. Note that $\boldsymbol{\eta}$ acute is incompatible with tetragonal symmetry.

c. REMARKS ON WULFF'S THEOREM.

Stable crystals $\Gamma$ are those that minimize total interfacial energy at fixed volume:

$$
\int_{\partial \Gamma} \hat{\gamma}(n) \leq \int_{\partial \Theta} \hat{\gamma}(n) \text { for all crystals } \Theta \text { with } \operatorname{vol}(\Theta)=C \text {, }
$$

with $C>0$ a prescribed constant (Wulff's Problem). This problem has a unique solution (modulo translation), and solutions for two different values of $\mathrm{C}$ differ by a dilation. Stable crystals have the following properties:

(W1) the orientation of each facet is admissible;

(W2) the orientations - of facets that intersect at an edge - are adjacent;

(W3) each set - of orientations of facets that intersect at a corner - is compatible.

We will refer to (W1)-(W3) as Wulff conditions; these conditions do not uniquely characterize stable crystals, since stable crystals are also, for example, bounded and convex, but we will find (W1)-(W3) useful as conditions to be imposed on crystalline motions. We remark that (5.7) is equivalent to the requirement that $\eta$ (and not a proper subset of $\pi$ ) be the set of orientations for any stable crystal.

The next proposition establishes, for crystalline motions consistent with the Wulff conditions, a one-to-one correspondence between possible crystal corners and faces on the Frank diagram.

Proposition 5.1. Let \& be a crystalline motion consistent with the Wulff conditions, let $\mathrm{c}$ be any corner of 8 , and let $\mathrm{I}$ denote the set of orientations of facets that intersect at $\mathrm{c}$. Then $\mathrm{In}$ is compatible and complete; thus there is a unique face

$$
f=\hat{f}(c)
$$

of the Frank diagram such that Frank $(\mathfrak{M})$ is the set of vertices of $f$. 
- Remark. The Wulff conditions; as general requirements on crystalline motions, are independent. Indeed, a single evolving facet has no corners or edges, so that (W1) is necessary. For \& an infinite wedge consisting of two facets intersecting along an edge, (W2) does not follow from (W1), and, since \& has no corners, (W3) is empty; hence (W2) is needed. Finally, if the Frank diagram has as its vertices $n_{1}=3^{-\frac{1}{2}}(1,1,1), n_{2}=3^{-\frac{1}{2}}(1,-1,1)$, $n_{3}=(-1,0,0), \quad n_{4}=(0,0,1), \quad n_{5}=(0,0,-1) ;$ if $\&$ consists of three unbounded facets meeting at a single corner; and if $n_{1}, n_{2}$, and $n_{3}$ are the orientations of these facets; then (W1) and (W2) are satisfied, but (W3) is not. 


\section{BASIC EQUATIONS. EXISTENCE}

a. DATA

We assume, as prescribed:

(i) a set $\eta$ of admissible orientations;

(ii) for each $n \in \Omega$, an interfacial free energy $\hat{\gamma}(n)>0$ and a kinetic modulus $\beta(n)>0$;

(iii) a (constant) bulk free energy $U$.

As before the terms "crystalline surface 8 " and "crystalline motion 8 " carry with them the requirement that the outward unit normal to \& be restricted to the set $\pi$ of admissible orientations, which is the Wulff condition (W1); consistency with the Wulff conditions (W2) and (W3) is not presumed.

\section{b. EVOLUTION EQUATIONS FOR CRYSTALLINE SURFACES}

The basic equations are the facet and edge balances (3.11) and (3.15), the thermodynamic identity (4.3), and the thermodynamically admissible constitutive relations (5.3) and (5.4). Granted (4.3), (5.3), (5.4) (and (3.12)), these are equivalent, not only to the original balance (3.1) with $\mathrm{C}$ defined through (3.6), but, more important, to the requirement that the evolution of each facet $F_{a}$ be consistent with the balance

$$
\operatorname{div}_{8} c=U+\beta\left(n_{a}\right) V
$$

and the edge condition

$$
c_{a} \cdot v_{a}=c_{a b}
$$

on each edge $(a, b)$ of $F_{a}$, where $c_{a b}$ are the constants defined by

$$
c_{a b}=\operatorname{sgn}\left(\nu_{a} \cdot n_{b}\right)\left[\left(n_{a} \cdot n_{b}\right) \hat{\gamma}\left(n_{a}\right)-\hat{\gamma}\left(n_{b}\right)\right] /\left[1-\left(n_{a} \cdot n_{b}\right)^{2}\right]^{\frac{1}{2}},
$$

while $v_{a}$ is the outward unit normal to $\partial F_{a}$. In view of the agreements made in Subsection $6 a$, the $c_{a b}$ may be viewed as prescribed data.

Let 


$$
\begin{aligned}
& L_{a b}(t)=\text { length of the }(a, b) \text { edge, } \\
& A_{a}(t)=\text { area of } F_{a}(t), \\
& V_{a}(t)=\text { normal velocity of } F_{a}(t), \\
& \beta_{a}=\beta\left(n_{a}\right) .
\end{aligned}
$$

Then, since the right side of $(6.1)$ is spatially constant on $F_{a}$, for $F_{a}$ bounded, if we integrate (6.1) over $F_{a}$ and use (6.2), we find that

$$
\beta_{a} V_{a}(t)=A_{a}(t)^{-1} \sum_{b} c_{a b} L_{a b}(t)-U
$$

where the sum is over all $b$ with $F_{b}$ adjacent to $F_{a}$ (cf. (3.15) and (4.3)). We write

$$
\Lambda_{a}(t)=A_{a}(t)^{-1} \sum_{b} c_{a b} L_{a b}(t) \text { for } F_{a} \text { bounded. }
$$

By (6.2), the constant $c_{a b}$ represents the shearing force exerted on $F_{a}$ by the facet $F_{b}$, so that $\Lambda_{a}(t)$ represents the net shear, per unit area, on $F_{a}$. We will refer to $\Lambda_{a}(t)$ as the energetically-weighted curvature (cf. Taylor [26], who uses the term weighted mean-curvature) and to the $c_{a b}$ as the curvature constants for the facet $F_{a}$.

By (6.2), there is a $C>0$, depending only on the set $\pi$ and the values of $\hat{\gamma}(n)$ for $n \in \mathbb{n}$ such that $\left|\Lambda_{a}\right| \leq C$ perimeter $\left(F_{a}\right) /$ area $\left(F_{a}\right)$. Bearing this in mind, and recalling our agreement to limit our discussion to crystals which, when unbounded, are consistent with (2.3), we adopt the convention:

$$
\Lambda_{a}(t) \equiv 0 \text { for } F_{a} \text { unbounded. }
$$

We are then led to the requirement that each facet $F_{a}(t)$, whether bounded or unbounded, evolve according to

$$
\beta_{a} V_{a}(t)=\Lambda_{a}(t)-U
$$

One may ask if (6.5)-(6.7) by themselves characterize crystalline motions consistent with the force balance (3.1); or equivalently (cf. the discussion surrounding (6.1) and (6.2)) whether, given a crystalline motion $\&$ 
consistent with (6.5)-(6.7), there is a tangential vector field $c$ on 8 that satisfies (6.1) and (6.2) on each facet. $F_{a}(t)$. Thus choose a facet $F_{a}(t)$ and a time $t$, and note that, by (6.7), (6.1) is equivalent to $\operatorname{div}_{s} c=\Lambda_{a}$ on $F_{a}$. To construct $c$, we assume, without loss in generality, that $\Omega_{a}=(0,0,1)$, so that $c$ has the form $\left(\eta_{1}, \eta_{2}, 0\right)$ with $\eta$ a planar vector field on the planar domain $F_{a}(t) \subset \mathbb{R}^{2}$; the determination of $c$ thus reduces to solving the boundary-value problem

$$
\begin{array}{ll}
\operatorname{div} \eta=\Lambda_{a} & \text { in the interior of } F_{a} \\
\eta \cdot \nu_{a}=c_{a b} & \text { on each edge }(a, b) \text { of } \partial F_{a} .
\end{array}
$$

The existence of a solution will be established in Lemmas 9.6 and 9.7 ; in fact, we will construct an $\eta$ that is piecewise linear (and bounded even when $F_{a}$ is unbounded). In this regard, note that, by (6.5), the "data" $\Lambda_{a}$ and $c_{a b}$ are compatible in the standard sense for Neumann boundary-conditions.

The solution $c$ is generally not unique, but this is no problem, as any two solutions yield the same crystalline motion. Given $c$, we can construct a stress field $\mathrm{C}$ via (3.6) with $\sigma$ given by (4.3) and (5.3); and, given $\mathrm{C}$, there is a Cahn-Hoffman vector $\boldsymbol{\xi}$, which is related to $\mathrm{C}$ through (3.17) and is consistent with (3.18) and (3.19). We will refer to $C$ and $\xi$ as $a$ capillary stress (field) and Cahn-Hoffman vector (field) for $\&$.

\section{c. EXISTENCE OF CRYSTALLINE MOTIONS}

We now discuss the existence of crystalline motions that start from a crystalline surface $\delta_{0}$ and evolve according to $(6.7)$ with $\eta, \hat{\gamma}(\cdot), \beta(\cdot)$, and $U$ as prescribed in Subsection $6 a$. Consistency with the Wulff conditions (W2) and (W3) is not required, although, by continuity, if the initial crystal $\delta_{0}$ is consistent with a Wulff condition (W), then any crystalline motion $\&$ with $\&(0)=\delta_{0}$ will be consistent with $(W)$.

We begin with some notation. Let $\delta_{0}$ be a crystalline surface with at least one corner. It is convenient to:

- label the facets of $s_{0}$ by $a=1,2 \ldots, Q$,

- label the corners of $s_{0}$ by $c=1,2 \ldots, e$,

- write $Q_{c}$ for the set of facet-labels for facets that meet at a corner $c$,

- write $e_{a}$ for the set of corner-labels for corners of a facet a.

Let $Y_{c}$ denote the position (vector) of an arbitrary corner $c$ of $\delta_{0}$, 
and let

$$
Y=\left(y_{1}, y_{2}, \ldots, y_{e}\right)
$$

denote the list of corner positions of $\delta_{0}$. By "slightly perturbing" $Y \in\left(\mathbb{R}^{3}\right) C$ we can find an open neighborhood $X(Y)$ of $Y$ in $\left(\mathbb{R}^{3}\right)^{\mathcal{C}}$ such that each $X=\left(x_{1}, x_{2}, \ldots, x_{l}\right) \in X(Y)$ is a list of corner positions of a polygonal surface Crystal $(X)$ each of whose facets corresponds-in an obvious manner-to a facet of $8_{0}$. This correspondence allows us to label the corners and facets of Crystal (X) using the corresponding labels of $\delta_{0}$. (Note that the outward unit normal to Crystal(X) will generally not have values in $\pi$.) Further, in the notation of Subsection $6 b$, for $X \in X(Y)$, each edge length $L_{a b}$ and each facet-area $A_{a}$ of $\operatorname{Crystal}(X)$ is a smooth function of $X$. The right side of $(6.7)$ is therefore a function $\Phi_{a}(X)$ on $X(Y)$ that is well defined and smooth, a function whose form depends only on $\beta, \hat{\gamma}(\cdot)$, and $U .\left(\Phi_{a}(X) \equiv-U\right.$ if the facet $a$ is unbounded.)

Next, let $\&$ be a crystalline motion starting from $\delta_{0}$, and, for any corner $c$ of 8 , let $\mathbf{x}_{c}(t)$ denote the position of $c$ at time $t$. Then the normal velocity $V_{a}(t)$ of each facet $a \in Q_{c}$ satisfies

$$
V_{a}=n_{a} \cdot\left(d x_{c} / d t\right)
$$

hence, for $t$ sufficiently small, the evolution equation (6.7) is equivalent to the requirement that, for each corner $c$,

$$
\beta_{a} n_{a} \cdot\left(d x_{c} / d t\right)=\Phi_{a}(X) \text { for all } a \in Q_{c} \text {, }
$$

where $X(t)=\left(x_{1}(t), x_{2}(t), \ldots, x_{e}(t)\right)$.

Suppose, for the moment, that each corner of $s_{0}$ is a triple junction; that is, each corner of $\delta_{0}$ is the intersection of exactly three facets. The set $\left\{n_{a}: a \in Q_{c}\right\}$ is then a basis for $\mathbb{R}^{3}$, and (6.10) may be solved for $\mathrm{dx}_{\mathrm{c}} / \mathrm{dt}$; hence there is a smooth mapping $\Phi$ of $X(Y)$ into $\left(\mathbb{R}^{3}\right)^{e}$ such that (6.10) (and thus (6.7)) is equivalent, for sufficiently small time, to an ordinary differential equation

$$
\mathrm{d} X / \mathrm{d} t=\Phi(X)
$$


for the list " $X(t)$ of corner positions of $B(t)$.

Suppose now that we are given a crystalline surface $s_{0}$ with each corner a triple junction, and we wish to find a crystalline motion $\&$ that satisfies $(6.7)$ and the initial condition $\delta(0)=\delta_{0}$. Assume that $\delta_{0}$ has at least one corner, let $Y$ denote the list of corner positions of $\delta_{0}$, and let $X(Y)$ be the open set defined above. Let $X(t)$ denote the unique (maximal) solution of (6.11) over $X(Y)$ subject to the initial condition $X(0)=Y$. Then $8(t)=$ Crystal $(X(t))$ will have the desired properties provided:

(1) the orientation of the facet $F_{a}(t)$ of $8(t)$ labelled a is equal to the

orientation $\mathrm{n}_{\mathrm{a}}$ of the corresponding facet of $\delta_{0}$;

(2) each facet of $8(t)$ evolves according to (6.7).

Consider (1). By (6.10), the projection on $n_{a}$ of the velocity of each corner $c$ of $F_{a}(t)$ is independent of $c$, and hence the orientation of $F_{a}(t)$ cannot vary with time; since the orientation of $F_{a}(0)$ is $n_{a},(1)$ is satisfied. On the other hand, (2) is a direct consequence of (6.9) and (6.10).

Finally, assume that $s_{0}$ has no corners, so that $s_{0}$ is the union of infinite facets. (Since these facets are regular, $\delta_{0}$ is either a plane or two half planes meeting at an edge.) Then (6.7) has the simple form $\beta_{a} V_{a}(t)=-U$ for each facet $a$, and the existence of a unique \& satisfying (6.7) and the initial condition $s(0)=s_{0}$ is immediate.

We therefore have an existence theorem for crystalline motions:

Theorem 6.1. Let $\delta_{0}$ be a crystalline surface with each corner a triple junction. Then there is a $\mathrm{T}>0$ and a unique crystalline motion \& with duration $\mathrm{T}$ such that:

(i) each facet of $\&$ evolves according to (6.7);

(ii) $s(0)=8_{0}$.

Suppose now that $\delta_{0}$ has a corner $c$ that is not a triple junction, and let $\delta(t)$ satisfy $(6.7)$ and the initial condition $s(0)=8_{0}$. Then for $Y$ the list of corner positions of $\delta_{0}$ and $v$ the velocity of the corner $c$ at $t=0,(6.10)$ yields

$$
\beta_{a} n_{a} \cdot v=\Phi_{a}(Y) \text { for all } a \in Q_{c}
$$


Since $Q_{c}$ has more than three elements, this represents an overdetermined system of equations for $v$, and will generally have no solution, an outcome that would render $8_{0}$ incompatible as initial data for a crystalline motion consistent with (6.7). Here we will not establish the most general conditions on the data that lead to nonexistence, but we will give a simple example.

Theorem 6.2. Let $s_{0}$ be a crystalline surface consisting of four unbounded facets, $a=1,2,3,4$, meeting at a single corner. Then there is a choice of $\beta\left(n_{a}\right)>0, a=1,2,3,4$, for which there is no crystalline motion \& that satisfies $\&(0)=\delta_{0}$ and $\beta_{a}\left(n_{a}\right) V_{a}=-U$ for $a=1,2,3,4$.

Proof. Geometry requires that the set $\left\{n_{a}: a \in Q_{c}\right\}$ have four elements. Further, (6.10) reduces to $\beta_{a} n_{a} \cdot v=-U$, and the equations for $a=1,2,3$, say, determine $v$. We can always choose $\beta_{4}>0$ so that the equation for $a=4$ is not satisfied.

Remarks.

(1) This failure of existence demonstrates that our framework is too narrow to include evolution from an initial crystal whose corners are not triple junctions; such initial data seems to require the formation of new edges. (Cf. the remark following Theorem 10.2, which notes that a crystalline surface can be approximated by a crystalline surface all of whose corners are triple junctions.) If global solvability is required it is necessary to allow for arbitrary initial data. For a smooth energy $\gamma$ a level set method ([8],[12]) is standard, but this method has not yet been developed for crystalline energies, although it should extend to crystalline graph-curves [14]; in this instance the solution seems to become a varifold (infinitesimally wrinkled) solution as constructed in [7] for mean curvature flows.

(2) For $\&_{0}$ a crystalline surface consisting of three unbounded facets, $a=1,2,3$, meeting at a single corner, the corresponding crystalline motion established in Theorem 6.1 is a uniform translation of $\delta_{0}$ with translational velocity $v$ the unique solution of $\beta_{a} n_{a} \cdot v=-U, a=1,2,3$. This motion exists for all time.

(3) For $s_{0}$ a crystalline surface consisting of two infinite facets meeting along a single infinite edge, the crystalline motion of Theorem 6.1 again consists of $s_{0}$ translating uniformly with velocity $v$. Here $v$ is any 
solution of $\beta_{a} n_{a} \cdot v=-U, a=1,2$, the component of $v$ perpendicular to $n_{1}$ and $n_{2}$ being tangential to $\&$ and of no importance.

(4) The convexity assumption on $F$ in Section $5 \mathrm{~b}$ is irrelevant to the validity of the results of this section. 


\section{SUBDIFFERENTIAL OF THE CONVEXIFIED ENERGY}

a. THE SUBDIFFERENTIAL

The convexified energy $\gamma_{0}(p)$ is not differentiable at points $p$ with $p /|p| \in \Omega$. With this in mind, we introduce the subdifferential

$$
\partial \gamma_{0}(p)=\left\{\xi \in \mathbb{R}^{3}: \gamma_{0}(p+h)-\gamma_{0}(p) \geq \xi \cdot h \quad \forall h \in \mathbb{R}^{3}\right\}
$$

of $\gamma_{0}$ at $p$; since $\gamma_{0}$ is convex, if $\gamma_{0}$ is differentiable at $p$, then

$$
\partial \gamma_{0}(p)=\left\{\nabla \gamma_{0}(p)\right\}
$$

Some useful properties of the convexified energy are, for $p \neq 0, \lambda>0$, $\boldsymbol{\xi} \in \partial \gamma_{0}(\mathbf{p})$,

$$
\begin{aligned}
& \gamma_{0}(\lambda p)=\lambda \gamma_{0}(p), \quad \partial \gamma_{0}(\lambda p)=\partial \gamma_{0}(p), \\
& \gamma_{0}(p)=\xi \cdot p .
\end{aligned}
$$

The first two relations in (7.3) follow from the definition (5.9) of $\gamma_{0}$. To establish the third we take $h=\alpha p,|\alpha|<1$, in the inequality in (7.1); this leads to $\alpha \gamma_{0}(p) \geq \alpha p \cdot \xi$, and, since the sign of $\alpha$ is arbitrary, this yields the desired result.

The next proposition relates the subdifferential to the Frank diagram $\mathcal{F}$. By a generalized tangent plane to $\mathcal{F}$ at $p \in \mathcal{F}$ we mean a plane $\pi$ through $p$ that does not intersect the set $\left\{z \in \mathbb{R}^{3}: \gamma_{0}(z)<1\right\}$ enclosed by $\mathcal{F}$.

Proposition 7.1. Let $p \in \mathcal{F}, \xi \in \mathbb{R}^{3}, \xi=0$. Then the following are equivalent:

(a) $\xi \in \partial \gamma_{0}(p)$.

(b) $\boldsymbol{\xi} \cdot \mathbf{p}=1$ and $\boldsymbol{\xi} \cdot(\mathbf{z}-\mathbf{p}) \leq 0$ for all $\mathbf{z} \in \mathcal{F}$.

(c) The plane $\Pi$ through $\mathrm{p}$ normal to $\xi$ is a generalized tangent plane to $\mathcal{F}$ at $\mathrm{p}, \boldsymbol{\xi}$ is directed outward from $\mathcal{F}(\boldsymbol{\xi} \cdot \mathrm{p}>0)$, and $|E|^{-1}$ is the perpendicular distance of $\Pi$ from the origin.

Proof. Assume that (a) is satisfied. Then, for $z \in \mathcal{F}$, (7.1) yields $\gamma_{0}(z)-\gamma_{0}(p) \geq \boldsymbol{\xi} \cdot(z-p)$, while $(5.10)$ implies $\gamma_{0}(z)=\gamma_{0}(p)=1$. Thus $\boldsymbol{\xi} \cdot(z-p) \leq 0$. 
Further, $(7.3)_{3}$ yields $\boldsymbol{E} \cdot \mathbf{p}=1$. Thus (b) holds. Conversely, assume that (b) is satisfied. Choose $r \in \mathbb{R}^{3}$. Then there is a scalar $\lambda>0$. such that $\lambda r \in \mathcal{F}$ and

$$
0 \geq \xi \cdot(\lambda r-p)=\lambda \xi \cdot(r-p)-(1-\lambda) .
$$

But by $(5.10), \gamma_{0}(p)=\lambda \gamma_{0}(r)=1$; hence $(7.4)$ yields

$$
0 \geq \xi \cdot(r-p)+\gamma_{0}(p)-\gamma_{0}(r) .
$$

Thus, since $r$ is arbitrary, $\xi \in \partial \gamma_{0}(p)$. Therefore $(a)$ and $(b)$ are equivalent.

Next, we show that $(b) \Leftrightarrow(c)$. Let $\Pi$ be the plane through $p$ normal to $\boldsymbol{\xi}$, and let $d$ denote the perpendicular distance of $\pi$ from the origin. Then the second assertion in (b) is equivalent to the requirement that $\Pi$ be a generalized tangent; and, since $\xi \cdot p>0$ implies $d=p \cdot \xi /|\xi|, \xi \cdot p=1$ if and only if $\boldsymbol{\xi} \cdot \mathbf{p}>0$ and $d=|\boldsymbol{\xi}|^{-1}$.

The energy $\gamma_{0}(p)$ is differentiable at all $p$ with $p /|p| \notin \mathfrak{N}$; in fact, by $(7.3)_{2}$ and the equivalence of (a) and (c) in the last proposition, given any face $f$ on the Frank diagram, $\nabla \gamma_{0}$ is constant on the cone

$$
e(f)=\{\alpha p: p \in f, p /|p| \notin \Omega, \alpha>0\}
$$

We denote this constant by $\nabla \gamma_{0}(f)$ :

$$
\nabla \gamma_{0}(f)=\nabla \gamma_{0}(p), \quad p \in \mathcal{C}(f) .
$$

The next lemma, whose proof follows from the equivalency of (a) and (c) in Proposition 7.1, will be useful.

\section{Lemma 7.1.}

(a) Let $\mathbb{M} \subset \boldsymbol{\eta}$. If $\mathfrak{M}$ is compatible and contains three or more vectors or two vectors that are not adjacent, then Frank $(\mathfrak{M})$ is contained in a unique face $f$ of $\mathcal{F}$ and

$$
\bigcap_{n \in \mathbb{M}} \partial \gamma_{0}(n)=\left\{\nabla \gamma_{0}(f)\right\} .
$$


If $\mathrm{In}$ is not compatible, then

$$
\bigcap_{n \in \mathbb{R}} \partial \gamma_{0}(n)=\varnothing .
$$

(b) If $\mathrm{m}, \mathrm{n} \in \boldsymbol{N}$ are adjacent orientations, so that the line segment from Frank $(m)$ to Frank $(n)$ is an edge of $F$, and if $f$ and $g$ are the faces of $\mathcal{F}$ that intersect along this edge, then

$$
\partial \gamma_{0}(m) \cap \partial \gamma_{0}(n)=\left\{\lambda \nabla \gamma_{0}(f)+(1-\lambda) \nabla \gamma_{0}(g): 0 \leq \lambda \leq 1\right\} .
$$

b. CHARACTERIZATION OF ADMISSIBLE CRYSTALLINE MOTIONS USING THE SUBDIFFERENTIAL. CAHN-HOFFMAN VECTOR

The next result shows that the Wulff conditions (W2) and (W3) may be stated in terms of the subdifferential of the energy.

Theorem 7.1. Let 8 be a crystalline motion consistent with (W1). Then:

(a) \& satisfies (W3) if and only if, for $\mathrm{c}$ any corner of 8 and $\mathfrak{M}$ the set of orientations of facets that intersect at $c$,

$$
\bigcap_{n \in \pi} \partial \gamma_{0}(n) \text { is a singleton; }
$$

(b) 8 satisfies (W2) if and only if: for $\mathrm{m}$ and $\mathrm{n}$ orientations of facets that intersect at an edge of 8 ,

$$
\partial \gamma_{0}(m) \cap \partial \gamma_{0}(n) \text { is a line segment. }
$$

Proof. To establish $(a) \Leftrightarrow(W 3)$ it suffices to show that if $c$ is a corner of $\&$ and $\mathbb{N}$ is the set of orientations of facets that intersect at $c$, then

$$
\text { In compatible } \Leftrightarrow(7.10)
$$

The assertion (7.12) follows from (a) of Lemma 7.1.

Similarly, (b) $\Leftrightarrow(W 2)$ will follow if we can show that: for $m$ and $n$ orientations of facets that intersect at an edge, 


$$
\mathrm{m}, \mathrm{n} \text { adjacent } \Leftrightarrow \cdot(7.11)
$$

The forward implication follows from $(\beta)$ of the Lemma. To establish the reverse implication, assume that (7.11) is satisfied, and suppose that $m$ and $\mathrm{n}$ are not adjacent. Let $\mathbb{N}=\{m, n\}$. If $\mathbb{N}$ is compatible, then (7.7) holds; if $\mathbb{M}$ is not compatible, then (7.8) holds; in either case (7.11) is not satisfied.

Theorem 7.2. Let \& be a crystalline surface consistent with (W1) and (W2). Let $\mathrm{F}_{\mathrm{a}}$ and $\mathrm{F}_{\mathrm{b}}$ be adjacent facets with $\mathrm{n}_{\mathrm{a}}$ and $\mathrm{n}_{\mathrm{b}}$ the corresponding orientations, and let $v_{a}$ be the outward unit normal to $\partial \mathrm{F}_{\mathrm{a}}$ on the $(\mathrm{a}, \mathrm{b})$-edge. Then

$$
\left[\partial \gamma_{0}\left(n_{a}\right) \cap \partial \gamma_{0}\left(n_{b}\right)\right] \cdot \nu_{a} \text { is the singleton }\left\{-c_{a b}\right\} \text {. }
$$

Further, let $f$ and 8 denote the faces of the Frank diagram that intersect along the line segment from Frank $\left(n_{a}\right)$ to Frank $\left(n_{b}\right)$. Then, for $h=f, g$ and $n=n_{a}, n_{b}$.

$$
\nabla \gamma_{0}(h) \cdot n=\gamma_{0}(n), \quad \nabla \gamma_{0}(h) \cdot v_{a}=-c_{a b} .
$$

Proof. Choose $h \in\{f, g\}$ and $n \in\left\{n_{a}, n_{b}\right\}$. By (7.3) 4 and (7.6),

$$
m \cdot \nabla \gamma_{0}(h)=\gamma_{0}(m), \quad \quad m \in S^{2} \cap e(h) ;
$$

thus, given any $m \in S^{2} \cap e(h)$ there is a vector $\hat{c}(m)$ such that

$$
\nabla \gamma_{0}(h)=\hat{\gamma}_{0}(m) m-\hat{c}(m), \quad \hat{c}(m) \cdot m=0,
$$

and, by the continuity of $\gamma_{0}(\mathrm{~m}),(7.17)$ and hence $(7.15)_{1}$ holds also for $\mathrm{m}=\mathrm{n}$.

Next, let $v_{a}$ and $\nu_{b}$ denote the outward unit normals to $\partial F_{a}$ and $\partial F_{b}$ on the edge $(a, b)$. Then $\nu_{a}$ and $\nu_{b}$ lie in the plane spanned by $n_{a}$ and $n_{b}$ with $v_{a} \times n_{a}=-v_{b} \times n_{b}$, and therefore 


$$
n_{a} \otimes v_{a}-v_{a} \otimes n_{a}=-\left[n_{b} \otimes \nu_{b}-v_{b} \otimes n_{b}\right]
$$

(the left side acting on a vector $v$ is $\pm\left(\nu_{a} \times n_{a}\right) \times v$; the right side is also, with the same sign). Thus applying the left side of (7.18) to (7.17) at $m=n_{a}$ and the right side at $m=n_{b}$, we conclude that (3.13) holds with $\sigma_{q}=\hat{\gamma}_{o}\left(n_{q}\right)$ and $c_{q}=\hat{c}\left(n_{q}\right), q=a, b$. Thus (3.15) and hence (6.2) is satisfied, and, since $\nabla \gamma_{0}(h) \cdot v_{a}=-c_{q} \cdot v_{a},(7.15)_{2}$ is also satisfied.

Finally (7.14) follows from (7.9) with $n=n_{a}$ and $m=n_{b}$ in conjunction with $(7.15)_{2}$.

The discussion in the last two paragraphs of Subsection $6 \mathrm{~b}$ ensures the existence of a Cahn-Hoffman vector $\boldsymbol{\xi}$ corresponding to 8 such that:

(a) on each facet,

$$
\operatorname{div}_{s}(P \xi)=\pi, \quad \xi \cdot n=\hat{\gamma}_{0}(n)
$$

(b) the projection of $\xi$ onto the plane perpendicular to each edge is continuous across the edge, or equivalently, $c=-P \xi$ is consistent with (6.2) on each edge.

Let $\&$ be a crystalline surface consistent (W1)-(W3). Given any corner $c$ of 8 , we refer to

$$
\xi_{0}(c)=\nabla \gamma_{0}(\hat{f}(c))
$$

as the Cahn-Hoffman vector for c (cf. (5.12)). Then, by (7.15), for c a corner terminating the $(a, b)$ edge,

$$
\xi_{0}(c) \cdot n_{a}=\gamma_{0}\left(n_{a}\right), \quad \xi_{0}(c) \cdot \nu_{a}=-c_{a b}
$$

Theorem 7.3. Let \& be a crystalline motion consistent with (W1). Then there is a piecewise linear Cahn-Hoffman vector $\boldsymbol{\xi}$ (which may not be continuous) corresponding to 8 that satisfies (a) (in a distributional sense) and (b). Further, for $\mathrm{T}$ the duration of 8 ,

(c). $\xi$ can be taken so that

$$
\xi(x, t) \in \partial \gamma_{0}(n(x, t))
$$


at a.e. $x$ of each facet of $\delta(t), t \in T$, if \& satisfies (W2). Conversely, given such a vector field $\boldsymbol{\xi}$, if $\boldsymbol{\xi}$ satisfies ( $\mathrm{a}$ ) and (b), then $8(t)$ satisfies (W2).

(d) if \& satisfies (W2) and $\boldsymbol{E}$ is the Cahn-Hoffman vector in (c), then $\&$ satisfies (W3) if and only if for any corner $c$ of \& there is a vector $\bar{\xi}$ independent of $b$ such that

$$
\xi(\mathbf{x}, t) \cdot \nu_{a}=\bar{\xi} \cdot v_{a}=-c_{a b}
$$

on each edge terminated by c. If (W3) holds, then $\bar{\xi}=\xi_{0}(c)$.

The converse assertions in (c) and (d) follow from Lemma 7.1(a); we postpone the proof of the remaining portions until Section 9 (cf. Theorem 9.8).

\section{c. ADMISSIBLE AND STRONGLY ADMISSIBLE CRYSTALLINE SURFACES}

In theories for evolving surfaces with smooth, convex interfacial energies the surface shear $c$ is not indeterminate, as it is here; instead $c$ is the derivative $c=\partial \hat{\gamma}(n) / \partial n$ of the interfacial energy on the unit sphere, or equivalently, the Cahn-Hoffman vector $\xi$ is the gradient $\xi=\nabla \gamma_{0}(n)$ on $\mathbb{R}^{3}$ of the convexified energy. Within the equilibrium theory these results are a consequence of Gibbs-stability $[9,22]$; within a dynamical framework they follow from thermodynamics $[2,20]$. The counterpart of $\xi=\nabla \gamma_{0}(n)$ within our theory is (7.22), a condition we believe is related to the stability of the crystal.

This discussion demonstrates the importance of the Wulff condition (W2) and motivates our introduction of a special terminology for surfaces with this property. By an admissible crystalline surface (or motion) \& we mean a crystalline surface (or motion) that is consistent with the Wulff conditions (W1) and (W2); if, in addition, 8 is consistent with (W3), then 8 is strongly admissible. (By Proposition 5.1, the set of orientations of facets that meet at a corner is complete if 8 is strongly admissible. The converse is trivial if $\&$ satisfies (W1) and (W2). The definition of strong admissibility given in the Introduction is therefore equivalent to that given here.) 
8. LOCAL REPRESENTATION OF THE INTERFACE AS A GRAPH

Throughout this section $\&$ is a crystalline surface, $F_{a}$ is a facet of $B$, and $\mathrm{n}_{\mathrm{a}}$ is the orientation of $F_{\mathrm{a}}$. Further, $P$ is the standard projection from $\mathbb{R}^{3}$ onto $\mathbb{R}^{2}$ :

$$
P(x)=\left(x_{1}, x_{2}\right), \quad x=\left(x_{1}, x_{2}, x_{3}\right) \in \mathbb{R}^{3}
$$

a. DEFINITION

We say that $\&$ is the graph of a function $u$ near $F_{a}$ if $u$ is a mapping from an open set $\Omega \subset \mathbb{R}^{2}$ into an open interval $I$ and, writing $\mathrm{D}=\Omega \times \mathrm{I}$ :

(G1) $D \cap \&=\left\{\mathbf{x} \in \mathbb{R}^{3}: \mathrm{x}_{3}=u\left(\mathrm{x}_{1}, \mathrm{x}_{2}\right),\left(\mathrm{x}_{1}, \mathrm{x}_{2}\right) \in \Omega\right\}$;

(G2) the closure of $\Omega_{a}=P\left(F_{a}\right)$ is contained in the interior of $\Omega=P(D)$;

(G3) $\Omega$ is a polygon, Dns consists of $F_{a}$ and portions of facets $F_{b}$ adjacent to $F_{a}, P\left(\partial F_{b}\right)$ intersects $\partial \Omega$ transversally, and $\Omega$ is bounded if $F_{a}$ is bounded.

In this case we will consistently use the notation:

$$
\begin{aligned}
& 8_{0}=8 \cap D=\text { graphlike portion of } \&, \\
& F_{b}=\text { an arbitrary facet adjacent to } F_{a}, \\
& \Omega_{a}=P\left(F_{a}\right), \\
& \Omega_{b}=P\left(F_{b}\right) \cap \Omega, \\
& n_{a}, n_{b}=\text { orientations of } F_{a} \text { and } F_{b}, \\
& |F|=\text { area of a two-dimensional set } F, \\
& |l|=\text { length of a line segment } l, \\
& \left.\ell_{a b}=\text { the (a,b)-edge (along which } F_{b} \text { intersects } F_{a}\right), \\
& \nu_{a}=\text { outward unit normal to } \partial F_{a} \text {, } \\
& \nu_{a b}=\text { outward unit normal to } \partial F_{a} \text { on } \ell_{a b}, \\
& \mu_{a b}=\text { outward unit normal to } \partial \Omega_{a} \text { on } P\left(l_{a b}\right) .
\end{aligned}
$$

We say that $\&$ is essentially a graph near $F_{a}$ if, modulo a rotation of the coordinates, $\&$ is the graph of some function $u$ near $F_{a}$. 


\section{Remarks.}

(1) (G3) is, in a.sense, superfluous: by suitably shrinking a set $\Omega$ consistent with (G1) and (G2), $\Omega$ can be made to satisfy (G3).

(2) If $n_{b} \cdot n_{a}>0$ for every $F_{b}$ adjacent to $F_{a}$, then $\&$ is essentially a graph near $F_{a}$.

(3) If 8 is consistent with (W1) and (W2) (so that $n_{a} \in \Omega$ ), and if $\pi$ is acute, then $n_{b} \cdot n_{a}>0$ for every $F_{b}$ adjacent to $F_{a}$; hence \& is essentially a graph near $F_{a}$.

b. IMPORTANT FORMULAE

Throughout this subsection $\&$ is the graph of a function $u$ near $F_{a}$. We now express the various geometrical and mechanical quantities associated with $\&$ in terms of their projections under $P$.

The areas of $F_{a}$ and $\Omega_{a}$ are related through

$$
\left|\Omega_{a}\right|=n_{3}\left|F_{a}\right|
$$

where $n=n_{a}=\left(n_{1}, n_{2}, n_{3}\right)$. Let $v=v_{a b}=\left(v_{1}, v_{2}, v_{3}\right)$. Then $v \times n$ is a unit tangent vector to $l_{a b}$, its projection

$$
\tau=\tau_{a b}=P(\nu \times n)=\left(\nu_{2} n_{3}-\nu_{3} n_{2}, \nu_{3} n_{1}-\nu_{1} n_{3}\right)=\left(\tau_{1}, \tau_{2}\right)
$$

is tangent to $P\left(l_{a b}\right)$, the lengths of $l_{a b}$ and $P\left(l_{a b}\right)$ satisfy

$$
\left|P\left(l_{a b}\right)\right|=|\tau|\left|l_{a b}\right|
$$

and $\mu=\mu_{a b}$ is given by

$$
\mu=|\tau|^{-1}\left(-\tau_{2}, \tau_{1}\right)
$$

It is convenient to introduce the mapping $\hat{q}$ from the upper unit hemisphere in $\mathbb{R}^{3}$ to $\mathbb{R}^{2}$ defined by

$$
\hat{q}(n)=-n_{3}^{-1}\left(p_{n}\right)
$$

then $\hat{q}$ is a bijection with inverse 


$$
\hat{n} \cdot(q)=(-. q, 1)\left(1+|q|^{2}\right)^{-1 / 2}
$$

and the normal $\mathrm{n}$ to $\delta_{0}$ is given, away from the edges of 8 , by

$$
\mathrm{n}=\hat{\mathrm{n}}(\nabla \mathrm{u})
$$

We now express the energy $\gamma_{0}(n)$, which is measured per unit area on $\delta_{0}$, as an energy $g(\nabla u)$ measured per unit area on $\Omega$; we define

$$
g(q)=\gamma_{0}(-q, 1), \quad q \in \mathbb{R}^{2}
$$

then, appealing to the homogeniety of $\gamma_{0}$,

$$
\int_{\delta_{0}} \gamma_{0}(n)=\int_{\Omega} \gamma_{0}(n)\left(1+|\nabla u|^{2}\right)^{1 / 2}=\int_{\Omega} g(\nabla u) .
$$

Since $\gamma_{0}$ is convex, so also is g; moreover,

$$
\partial g(q)=-P\left(\partial \gamma_{0}(-q, 1)\right)
$$

Further, $\nabla g(q)=-p \nabla \gamma_{0}(-q, 1)$ whenever $\nabla \gamma_{0}(-q, 1)$ exists; in fact, by (7.6), given any face $f$ on the Frank diagram, $\nabla g$ has the constant value $-P \nabla \gamma_{0}(f)$ on the polygonal region

$$
e^{*}(f)=\left\{q \in \mathbb{R}^{2}:(-q, 1) \in e(f)\right\}
$$

Thus

$$
\nabla g(f):=-p \nabla \gamma_{0}(f)=\nabla g(q) \quad \text { for all } q \in e^{*}(f)
$$

which is a counterpart of the relation (7.6).

Note that, for each unit vector $n$,

$$
n \in e(f) \Leftrightarrow \hat{q}(n) \in e^{*}(f)
$$

If $m, n \in \mathbb{N}$ are adjacent, then two faces $f$ and $g$ of the Frank diagram 
intersect along the line segment from Frank(m) to Frank(n), and the intersection of the polygonal regions $e^{*}(f)$ and $e^{*}(g)$ is the line segment from $\hat{q}(m)$ to $\hat{q}(n)$.

Let $F_{a}$ and $F_{b}$ be adjacent facets, write $n_{a}=\left(n_{1}, n_{2}, n_{3}\right)$, $v_{a b}=\left(\nu_{1}, v_{2}, \nu_{3}\right)$, and define constants $\tilde{c}_{a b}$ through

$$
\left|\tau_{a b}\right| \tilde{c}_{a b}=n_{3} c_{a b}+\nu_{3} \gamma_{0}\left(n_{a}\right)
$$

The next result is a direct analog of Theorem 7.2.

Theorem 8.1. Let 8 be a crystalline surface satisfying (W1) with $S_{0}$ the graph of a function near $F_{a}$. Let $F_{b}$ be a facet adjacent to $F_{a}$; let $\mathrm{n}_{\mathrm{a}}$ and $\mathrm{n}_{\mathrm{b}}$, assumed adjacent, denote the orientations of $\mathrm{F}_{\mathrm{a}}$ and $F_{b}$; let $q_{a}=\hat{q}\left(n_{a}\right), q_{b}=\hat{q}\left(n_{b}\right)$; and let $f$ and $g$ denote the faces of the Frank diagram that intersect along the line segment from Frank $\left(n_{\mathrm{a}}\right)$ to $\operatorname{Frank}\left(n_{b}\right)$. Then, for $h=f, g$,

$$
\begin{aligned}
& \nabla g(h) \cdot \mu_{a b}=\tilde{c}_{a b}, \\
& {\left[\partial g\left(q_{a}\right) \cap \partial g\left(q_{b}\right)\right] \cdot \mu_{a b}=\partial g\left(q_{a}\right) \cdot \mu_{a b} \cap \partial g\left(q_{b}\right) \cdot \mu_{a b}=\left\{\tilde{c}_{a b}\right\} .}
\end{aligned}
$$

Proof. An argument analogous to that used to establish (7.9) yields the conclusion

$$
\partial g\left(q_{a}\right) \cap \partial g\left(q_{b}\right)=\{\lambda \nabla g(f)+(1-\lambda) \nabla g(g): 0 \leq \lambda \leq 1\}
$$

Thus to establish $(8.15)_{1}$ and the identity $\left.\log \left(q_{a}\right) \cap \partial g\left(q_{b}\right)\right] \cdot \mu_{a b}=\left\{\tilde{c}_{a b}\right\}$, it suffices to show that

$$
c_{a b}=\left|\tau_{a b}\right| n_{3}^{-1} \mu_{a b} \cdot \nabla g(h)-\nu_{3} \gamma_{0}\left(n_{a} / n_{3}\right)
$$

For convenience, we write $e=e(h), e^{*}=e^{*}(h), \quad \eta=\nabla g(h), \xi=\nabla \gamma_{0}(h)$, $e=(0,0,1)$. In view of the remarks containing $(7.5), E=\nabla \gamma_{0}(m)$ for every $m$ in the interior of $C$. Thus, by (8.12),

$$
\boldsymbol{E} \cdot v_{\mathrm{ab}}=-v_{1} \eta_{1}-v_{2} \eta_{2}+v_{3} e \cdot \nabla \gamma_{0}(\mathrm{~m})
$$


for all such $m$. Further, since $\gamma_{0}$ is homogeneous, $\gamma_{0}(m)=m \cdot \nabla \gamma_{0}(m)$; hence (8.12) yields

$$
m_{3} e \cdot \nabla \gamma_{0}(m)=\gamma_{0}(m)+m_{1} \eta_{1}+m_{2} \eta_{2}
$$

therefore

$$
\xi \cdot v_{a b}=-v_{1} \eta_{1}-v_{2} \eta_{2}+v_{3} m_{3}^{-1}\left[\gamma_{0}(m)+m_{1} \eta_{1}+m_{2} \eta_{2}\right]
$$

Letting $m \rightarrow n_{a}$ from $e$ (which is equivalent to letting $\hat{q}(m) \rightarrow q_{a}$ from $\left.e^{*}\right)$ and rearranging terms, we find that

$$
\xi \cdot v_{a b}=v_{3} \gamma_{0}\left(n / n_{3}\right)+n_{3}^{-1}\left[\eta_{1}\left(n_{1} \nu_{3}-\nu_{1} n_{3}\right)+n_{2}\left(n_{2} v_{3}-v_{2} n_{3}\right)\right]
$$

By (7.15), $c_{a b}=-\xi \cdot \nu_{a b}$; thus (8.2), (8.4), and (8.21) yield (8.17).

It remains to show that

$$
\left[\partial g\left(q_{a}\right) \cap \partial g\left(q_{b}\right)\right] \cdot \mu_{a b}=\partial g\left(q_{a}\right) \cdot \mu_{a b} \cap \partial g\left(q_{b}\right) \cdot \mu_{a b}
$$

Note that $q_{a}-q_{b}$ is parallel to $\mu_{a b}$ and that, for $\partial G$ the subdifferential of $G(\sigma)=g\left(q_{a}+\sigma\left(q_{b}-q_{a}\right)\right)$

$$
\partial g\left(q_{a}\right) \cdot \mu_{a b}=\partial G(0), \quad \partial g\left(q_{b}\right) \cdot \mu_{a b}=\partial G(1)
$$

Since $G$ is convex and linear on $[0,1]$, the intersection of $\partial G(0)$ and $\partial G(1)$ is a singleton (and is, in fact, the derivative of $G$ in $[0,1]$ ). Finally, since

$$
\left\{\tilde{c}_{a b}\right\}=\left[\partial g\left(q_{a}\right) \cap \partial g\left(q_{b}\right)\right] \cdot \mu_{a b} \subset \partial g\left(q_{a}\right) \cdot \mu_{a b} \cap \partial g\left(q_{b}\right) \cdot \mu_{a b}=\partial G(0) \cap \partial G(1),
$$

the desired conclusion (8.22) follows.

The crucial ingredient in the evolution equation (6.7) is the energetically-weighted curvature $\Lambda_{a}(t)$ defined in (6.5). The next theorem, the main result of this section, gives this "curvature" in terms of quantities associated with motion as a graph. 
Theorem.. 8.2. Let 8 be an admissible crystalline surface represented as the graph of a function near a facet $F_{a}$, with $F_{a}$ bounded. Let the subscript $\mathrm{b}$ label the facets adjacent to $\mathrm{F}_{\mathrm{a}}$. Then the energetically-weighted curvature $\Lambda_{\mathrm{a}}$ is given by

$$
\Lambda_{a}=\left|\Omega_{a}\right|^{-1} \sum_{b} \tilde{c}_{a b}\left|p\left(l_{a b}\right)\right| .
$$

Proof. Let $v$ denote the outward unit normal to $\partial F_{a}$, so that, in terms of the notation used above, $\nu_{3}=e \cdot v=e \cdot v_{a b}$ on the $(a, b)$-edge. By (2.4) the projection $h=P\left(n_{a}\right) e$ of $e$ onto $F_{a}$ is constant and thus, since $e \cdot v=h \cdot v$, the divergence theorem applied on the facet $F_{a}$ yields the identity

$$
\sum_{b} e \cdot v_{a b}\left|l_{a b}\right|=\int_{\partial F_{a}} \nu_{3}=\int_{\partial F_{a}} e \cdot v=0
$$

In the current notation the relation (6.5) has the form

$$
\Lambda_{a}=\left|F_{a}\right|^{-1} \sum_{b} c_{a b}\left|l_{a b}\right|
$$

and therefore, applying (8.14) in conjunction with (8.1), (8.3), and (8.26), we are led to (8.25). 
9. VARIATIONAL FORMULATION

a. INTRODUCTORY REMARKS

Our next step is to express the evolution equation

$$
\dot{\beta}_{a} V_{a}(t)=\Lambda_{a}(t)-U
$$

for bounded facets $F_{a}$ in terms of the subdifferential of the total freeenergy

$$
U \text { vol } \Gamma(t)+\int_{\delta} \gamma_{0}(n)
$$

Here $\Lambda_{a}$ is the energetically-weighted curvature (6.5) (or (8.25)).

If $\delta$ is a crystalline surface represented as the graph of a function $u$ near $F_{a}$, then, in the notation of Section 8 , and using (8.9), we can associate an energy

$$
\Phi(w)=\int_{\Omega}[g(\nabla w)+U w]
$$

with the graphlike portion $\delta_{0}$ of $\delta_{;}$modulo a constant, $\Phi(w)$ represents the free energy of the interface when perturbed over $\delta_{0}$; that is, the free energy the interface would have were the graph $u$ replaced by $w$ on $\Omega$, where $w: \Omega \rightarrow \mathbb{R}$ with $w=u$ on $\partial \Omega$. For each $w$ the subdifferential $\partial \Phi(w)$ is a subset of functions on $\Omega$, and, as the main result of this section, we show that there is a "canonical element" $f \in \partial \Phi(u)$ such that $f=-\Lambda_{a}+U$ on $\Omega_{a}$, provided that $\&$ is admissible. We now make these ideas precise.

b. SUBDIFFERENTIALS IN HILBERT SPACE. TRACE LEMMA

Let $\Omega \subset \mathbb{R}^{n}$ be a bounded domain with Lipschitz boundary, let $j$ be a continuous convex function on $\mathbb{R}^{n}$, with $j$ coercive in the sense that

$$
\lim _{|q| \rightarrow \infty} j(q) /|q|=\infty,
$$

and let $H$ denote the Hilbert space $H=L^{2}(\Omega)$ with standard inner product 
$\langle\cdot$,$\rangle . Then for prescribed functions U \in H, \quad \psi \in \operatorname{Lip}(\partial \Omega)$, we consider the functional $\Phi$. defined on $H \cdot$ by

$$
\begin{aligned}
& \Phi(w)=\int_{\Omega}[j(\nabla w)+U w] \text { if } \nabla w \in\left[L^{1}(\Omega)\right]^{n}, j(\nabla w) \in L^{1}(\Omega),\left.w\right|_{\partial \Omega}=\psi \\
& \infty \text { otherwise; }
\end{aligned}
$$

and we let

$$
\partial \Phi(w)=\{f \in H: \Phi(w+h)-\Phi(w) \geq\langle h, f\rangle \text { for all } h \in H\} .
$$

Lemma 9.1. The functional $\Phi$ is convex and lower semicontinuous on $H$, and $\Phi \not \infty$. Further, given $f, w \in H$, the following are equivalent:

(a) $f \in \partial \Phi(w)$,

(b) $f=-\operatorname{div} \eta+U$ for some $\eta \in\left[L^{1}{ }_{l o c}(\Omega)\right]^{n}$ with $\eta(x) \in \partial j(\nabla w(x))$ for a.e. $\mathbf{x} \in \Omega$.

The convexity of $\Phi$ is not difficult to verify; the lower semicontinuity follows from an application of the Dunford-Pettis Theorem as in [6]. The assertion of equivalency, which is far more difficult to prove, can be obtained by a suitable modification of a proof found in [1, Theorem 2.15], where the underlying functional $\Psi$ is defined as follows: extend $\psi$ to $\Omega$ so that $\psi \in \mathrm{H} \cap \mathrm{W}^{1,1}(\Omega)$; then $\Psi(v)=\Phi(W)$ with $v=W-\psi \in H \cap W^{1,1}(\Omega)$ restricted by $\Phi(v)<\infty$. (Note that the continuity of $j$ is required by the proof.)

If $n=1$ and $U \equiv 0$, the characterization (b) of $\partial \Phi$ can be proved for lower semicontinuous $j: \mathbb{R} \rightarrow(-\infty, \infty)$ under periodic boundary conditions by adopting an idea of Brezis [6] (cf. [13]). We do not know whether such a characterization of $\partial \Phi$ holds for general lower semicontinuous convex and coercive $j(\not \infty)$, even when $U$ and $\psi$ vanish identically.

Lemma 9.2. Let $\Omega^{\prime} \subset \mathbb{R}^{\mathrm{n}}$ be a bounded domain with Lipschitz boundary. Suppose that $\Omega^{\prime}$ is the disjoint union of $\Omega^{+}, \Omega^{-}$, and $\Sigma$, with $\Omega \pm$ subdomains of $\Omega^{\prime}$ and $\Sigma$ a Lipschitz hypersurface such that the portions of $\partial \Omega^{+}$and $\partial \Omega^{-}$in the interior of $\Omega$ each coincide with $\Sigma$. Let $v$ denote the unit normal on $\Sigma$ directed outward from $\partial \Omega$. Suppose that $\zeta \in\left[\mathrm{L}^{2}\left(\Omega^{\prime}\right)\right]^{n}$ with $\operatorname{div} \zeta \in \mathrm{L}^{2}\left(\Omega^{\prime}\right)$. Then the normal trace 
$\operatorname{tr} \pm(\boldsymbol{\zeta} \cdot \boldsymbol{v})$ from $\Omega^{ \pm}$belongs to $\mathrm{H}^{-1 / 2}(\Sigma)$ and $\operatorname{tr}^{+}(\boldsymbol{\zeta} \cdot \boldsymbol{v})=\operatorname{tr}^{-}(\boldsymbol{\xi} \cdot \boldsymbol{v})$. If is bounded, i.e. $\boldsymbol{\zeta} \in\left(\mathrm{L}^{\infty}(\Omega)\right)^{n}$, then $\operatorname{tr} \pm(\zeta \cdot v) \in \mathrm{L}^{\infty}(\Sigma)$.

Proof. By localizing the problem and regarding $\Sigma$ as the graph of a Lipschitz function, we may assume that $\Omega^{\prime}=\mathbb{R}^{n}$ and that $\Sigma$ is the plane $\left\{x_{n}=0\right\}$. We may also assume that 5 has compact support. Since $\boldsymbol{\zeta} \in\left[\mathrm{L}^{2}\left(\mathbb{R}^{n}\right)\right]^{n}$ with $\operatorname{div} \boldsymbol{\xi} \in \mathrm{L}^{2}\left(\mathbb{R}^{n}\right)$, if we write $\mathbf{x} \in \mathbb{R}^{n}$ in the form $\left(x^{1}, x_{n}\right)$, then

$$
\rho^{ \pm}\left(x^{\prime}, \varepsilon\right):=(\boldsymbol{\zeta} \cdot \boldsymbol{v})\left(\mathbf{x}^{\prime}, \pm \varepsilon\right)
$$

as a function of $\mathbf{x}^{\prime}$, is well defined and bounded in $H^{-1 / 2}\left(\mathbb{R}^{n-1}\right)$ uniformly for $\varepsilon \geq 0$ (cf. [27]). Further, since $\rho^{ \pm}$is compactly supported, $\rho^{ \pm}\left(\mathbf{x}^{\prime}, \varepsilon\right) \rightarrow p^{ \pm}\left(\mathbf{x}^{\prime}, 0\right)$ in $H^{-(1 / 2)-\delta}\left(\mathbb{R}^{n-1}\right)$ for any $\delta>0$. Thus for $\varphi \in C_{0}{ }^{\infty}\left(\mathbb{R}^{n}\right)$, letting $A( \pm \varepsilon)=\left\{x: \pm x_{n}>\varepsilon\right\}$,

$$
\begin{aligned}
& \int_{\mathbb{R}^{n}} \varphi \operatorname{div} \boldsymbol{\xi}=\lim _{\varepsilon \rightarrow 0}\left[\int \varphi \operatorname{div} \boldsymbol{\xi}+\int_{A(+\varepsilon)} \varphi \operatorname{div} \boldsymbol{\zeta}\right] \\
& \quad=\int_{\mathbb{R}^{n}} \nabla \varphi \cdot \boldsymbol{\xi}+\lim _{\varepsilon \rightarrow 0} \int_{\mathbb{R}^{n-1}}\left[\left(\rho^{+}\left(\mathbf{x}^{\prime}, \varepsilon\right) \varphi\left(\mathbf{x}^{\prime}, \varepsilon\right)-\rho^{-}\left(\mathbf{x}^{\prime}, \varepsilon\right) \varphi\left(\mathbf{x}^{\prime}, \varepsilon\right)\right] \mathrm{d} \mathbf{x}^{\prime} .\right.
\end{aligned}
$$

But

$$
\int_{\mathbb{R}^{n}} \varphi \operatorname{div} \zeta=\int_{\mathbb{R}^{n}} \nabla \varphi \cdot \xi
$$

hence

$$
\int_{\mathbb{R}^{n-1}}\left[\left(p^{+}\left(\mathbf{x}^{\prime}, 0\right) \varphi\left(\mathbf{x}^{\prime}, 0\right)-\rho^{-}\left(\mathbf{x}^{\prime}, 0\right) \varphi\left(\mathbf{x}^{\prime}, 0\right)\right] \mathrm{d} \mathbf{x}^{\prime}=0\right.
$$

Since $\varphi \in C^{\infty}\left(\mathbb{R}^{n}\right)$, this yields the desired result $p^{+}\left(x^{\prime}, 0\right)=p^{-}\left(x^{\prime}, 0\right)$. If $\boldsymbol{\zeta} \in\left(\mathrm{L}^{\infty}(\Omega)\right)^{\text {n }}$, so that $\rho^{ \pm}\left(\mathbf{x}^{\prime}, \varepsilon\right)$ is bounded, then its weak* limit agrees with $\rho \pm(x, 0)$, independent of the choice of subsequence, as $\varepsilon \rightarrow 0$. Thus $P^{ \pm}(x, 0) \in L^{\infty}(\Sigma)$.

c. SUBDIFFERENTIAL OF THE TOTAL ENERGY

Throughout this section $\&$ is an admissible crystalline motion with $s\left(t_{0}\right)$ the graph of a function $u$ near $F_{a}$. We assume that $F_{a}$ is 
bounded, so that $\Omega$ is bounded. (Cf. (G3); here and in what follows, we use the notation of Section 8.) Since \& satisfies (W1), there is a Lipschitz constant $\mathrm{K}$ for $\mathrm{u}$ :

$$
|\nabla u(x)| \leq K \quad \text { for all } \mathbf{x} \in \Omega \text {. }
$$

We let $\mathrm{j}$ denote a coercive, convex, continuous function on $\mathbb{R}^{2}$ with the property that

$$
j(q)=g(q) \text { for }|q| \leq K+1
$$

(such a function exists). In addition, we let

$$
\psi=\left.u\right|_{\partial \Omega}
$$

so that, by (G3), $\psi$ is Lipschitz continuous. Finally, we define $\Phi$ on $H=L^{2}(\Omega)$ by (9.5) with this choice of $j$ and $\psi$, and with $U$ the constant bulk free-energy of the crystal.

We now show that the subdifferential $\partial \Phi(u)$ has a "canonical element" that coincides with $-\Lambda_{a}+U$ on $\Omega_{a}$. Precisely, we look for an element $f \in \partial \Phi(u)$ with the property that

$$
\|f\| \leq\|h\| \text { for all } h \in \partial \Phi(u) \text {. }
$$

Since $\Phi$ is lower semicontinuous and convex with $\Phi \neq \infty,-\partial \Phi$ becomes a maximal monotone operator and $\partial \Phi(u)$ is a convex closed set in $H$ for fixed $u$. Thus (9.10) has a unique solution $f$, which we write as $f=\partial^{\circ} \Phi(u)$; we will refer to $\partial^{\circ} \Phi(u)$ as the canonical subdifferential of $\Phi$ at $u$. The next result is the main result of this section; the proof requires only that $s(t)$ satisfy (W1) and (W2) at $t=t_{0}$.

Theorem 9.1. Assume that $\&$ is an admissible crystalline surface represented as the graph of a function $u$ near $F_{a}$. Then the canonical subdifferential $\partial^{\circ} \Phi(u)$ is constant on $\Omega_{a}=P\left(F_{a}\right)$ and is related to the energetically-weighted curvature $\Lambda_{a}$ and the bulk energy $U$ through 


$$
\partial^{\circ} \Phi(u)(x)=-\Lambda_{a}+U \quad \text { for } \mathbf{x} \in \Omega_{\mathbf{a}} \text {. }
$$

Proof. We first note that, by (9.7) and (9.8),

$$
j(\nabla u)=g(\nabla u) \text { on } \Omega .
$$

Further, since $\Omega$ is the union of a finite number of polygons with $\nabla u$ constant on each, functions $\boldsymbol{b}$ with $\boldsymbol{b}(\mathbf{x}) \in \partial g(\nabla u(x))$ for a.e. $\mathbf{x} \in \Omega$ belong to $\left[L^{\infty}(\Omega)\right]^{2}$. It is convenient to define, for $\Theta \subset \Omega$,

$$
G(\Theta)=\left\{\zeta \in\left[L^{\infty}(\Theta)\right]^{2}: \zeta(x) \in \partial g(\nabla u(x)) \text { for a.e. } x \in \Theta\right\} \text {. }
$$

Lemma 9.3. Let $\mathrm{f} \in \mathrm{H}$.

(i) $\mathrm{f} \in \partial \Phi(u)$ if and only if

$$
f=-\operatorname{div} \zeta+U \text { for some } \zeta \in G(\Omega) \text {. }
$$

(ii) Granted (9.14), the normal trace $\operatorname{tr}\left(\boldsymbol{\zeta} \cdot \mu_{\mathrm{ab}}\right)$ from $\Omega_{\mathrm{a}}$ agrees with $\tilde{c}_{a b}$ on each of the edges $p l_{a b}$ of $\Omega_{a}$.

Proof (Lemma). Assertion (i) follows from Lemma 9.1 and (9.12). Next, Lemma 9.2 yields $\operatorname{tr}_{a}\left(\boldsymbol{\xi} \cdot \mu_{a b}\right)=\operatorname{tr}_{b}\left(\boldsymbol{r} \cdot \mu_{a b}\right)$ (as elements of $L^{\infty}\left(\partial \Omega_{a}\right)$ ) on each edge $P\left(l_{a b}\right)$ of $\Omega_{a}$, where $\operatorname{tr}_{c}$ is the trace from $\Omega_{c}, c=a, b$. Let $n_{a}$ and $n_{b}$ denote the orientations of $F_{a}$ and $F_{b}$, and let $q_{a}=\hat{q}\left(n_{a}\right)$, $q_{b}=\hat{q}\left(n_{b}\right)$. Then $\boldsymbol{b}(x) \in \partial g\left(q_{a}\right)$ for $x \in \Omega_{a}$ and $\boldsymbol{r}(\mathbf{x}) \in \partial g\left(q_{b}\right)$ for $x \in \Omega_{b}$; hence

$$
\operatorname{tr}_{a}\left(\xi \cdot \mu_{a b}\right) \in \partial g\left(q_{a}\right) \cdot \mu_{a b} \cap \partial g\left(q_{b}\right) \cdot \mu_{a b}
$$

and (8.15) yields the conclusion $\operatorname{tr}_{a}\left(\boldsymbol{\zeta}: \mu_{a b}\right)=\tilde{c}_{a b}$.

Our next step shows that solutions 5 of (9.14) with $f=$ const. on $\Omega_{a}$ have $\operatorname{div} \boldsymbol{\xi}=\Lambda_{\mathrm{a}}$ on $\Omega_{\mathrm{a}}$.

Lemma 9.4. Suppose that $\zeta \in\left[L^{\infty}(\Omega)\right]^{2}$ and 


$$
\begin{array}{ll}
\operatorname{div} \zeta=\text { const. } & \text { on } \Omega_{a_{1}} \\
\boldsymbol{\zeta} \cdot \mu_{\mathrm{ab}}=\tilde{c}_{\mathrm{ab}} & \text { on each edge } P l_{a b} \text { of } \Omega_{\mathrm{a}} .
\end{array}
$$

Then

$$
\operatorname{div} \xi=\Lambda_{a} \quad \text { on } \Omega_{a}
$$

Proof (Lemma). We simply apply the divergence theorem to the integral of div over $\Omega_{a}$, and then use (9.16) and (8.25).

We now investigate the canonical subdifferential $\partial^{\circ} \Phi(u)$, which, by Lemma $9.3(i)$ has the form

$$
\partial^{\circ} \Phi(u)=-\operatorname{div} \eta+U, \quad \eta \in G(\Omega) .
$$

By Lemma 9.4, to complete the proof we have only to show that $\partial^{\circ} \Phi(u)$ is constant on $\Omega_{a}$. The next result shows that $\partial^{\circ} \Phi(u)$ has this property provided condition (*) below is satisfied. In stating $(*)$ note that $\nabla u=q_{a}$ on $\Omega_{a}$ with $q_{a}=\hat{q}\left(n_{a}\right)$.

(*) given any $\Theta$ whose closure is compact in $\Omega_{a}, n(x)$ is uniformly away from the boundary of $\partial g\left(q_{a}\right)$ for $x \in \Theta$.

Lemma 9.5. Let $\eta$ in (9.18) satisfy $(*)$. Then

$$
\partial^{\circ} \Phi(u)=\text { const. on } \Omega_{a} .
$$

Proof (Lemma). By Lemma 9.3(i) and the definition of $\partial^{\circ} \Phi(u), \eta$ minimizes $\|\operatorname{div} \boldsymbol{\zeta}-U\|$ over all $\zeta \in G(\Omega)$. Choose $\varphi \in\left[C_{0}^{\infty}\left(\Omega_{a}\right)\right]^{2}$. Then, by $(*)$,

$$
\eta+\varepsilon \varphi \in G(\Omega)
$$

for all sufficiently small $\varepsilon$. Thus, since $\eta$ is a minimizer,

$$
0=\left.(d / d \varepsilon)\right|_{\varepsilon=0} \int_{\Omega_{a}}[\operatorname{div}(\eta+\varepsilon \varphi)-U]^{2}=-2 \int \varphi \cdot \nabla[\operatorname{div} \eta-U],
$$

and the desired conclusion (9.19) follows, since $\varphi$ is arbitrary. 
The final step in the proof is to show that (9.18) has a solution $\eta$ satisfying (*). To accomplish this we first show that $\eta$ in (9.18) can be chosen to be piecewise linear on $\Omega_{a}$.

\section{Lemma 9.6.}

(a) There is a piecewise linear minimizer $\tilde{n}$ (satisfing $(*)$ ) of

$$
\int[\operatorname{div} \zeta-U]^{2}
$$

$\Omega_{a}$

over all $\zeta \in \mathrm{G}\left(\Omega_{\mathrm{a}}\right)$ that satisfy the boundary condition

$$
\xi \cdot \mu_{a b}=\tilde{c}_{a b} \text { on each edge } P l_{a b} \text { of } \Omega_{a} \text {. }
$$

(b) Let $\eta$ satisfy (9.18), let $\tilde{\eta}$ be as in (a), and define $\boldsymbol{\xi} \in \mathrm{G}(\Omega)$ through

$$
\begin{array}{ll}
\boldsymbol{\zeta}(\mathbf{x})=\tilde{\eta}(\mathbf{x}), & \mathbf{x} \in \bar{\Omega}_{\mathbf{a}}, \\
\boldsymbol{\zeta}(\mathbf{x})=\eta(\mathbf{x}), & \mathbf{x} \in \Omega-\bar{\Omega}_{\mathbf{a}} .
\end{array}
$$

Then

$$
\partial^{\circ} \Phi(u)=-\operatorname{div} \zeta+U \text { on } \Omega \text {, }
$$

so that (9.18) has a solution $\eta$ satisfing $(*)$.

Proof (Lemma).

(a) Let $q_{1}, q_{1}, \ldots, q_{k}$ denote the corners of $\Omega_{a}$, listed in counterclockwise order, and let $\mu_{j}(1 \leq j \leq k)$ denote the outward unit normal of the segment $q_{j} q_{j+1}$ with the agreement $q_{k+1}=q_{1}$. Then each segment $q_{j} q_{j+1}$ is an edge $P l_{a b}$ of $\Omega_{a}$ with $\mu_{j}=\mu_{a b}$; for convenience, we write $\tilde{c}_{j}=\tilde{c}_{a b}$. Let $Q$ be a polygon with corners $\bar{q}_{1}, \bar{q}_{1}, \ldots, \bar{q}_{k}$ such that the outward unit normal to $\bar{q}_{j} \bar{q}_{j+1}$ is $\mu_{j}$ and such that $\bar{q}_{j} \bar{q}_{j+1}$ is contained in the line $\boldsymbol{\zeta} \cdot \mu_{j}=\tilde{c}_{j}$. Then, since \& is admissible, we may conclude from Theorem 8.1 that $\partial g\left(\nabla u\left(\Omega_{a}\right)\right)=Q$, so that $Q$ is convex. 
Next, by Lemma 9.4 and the proof of Lemma 9.5, minimizers of

$$
\int_{\Omega}[\operatorname{div} \zeta-U]^{2}
$$

subject to (9.20) satisfy (9.17).

It now suffices to prove that there is a piecewise-linear function $\tilde{\eta} \in\left[L^{\infty}(\Omega)\right]^{2}$ satisfying (9.16) and (9.17) (with $\boldsymbol{\zeta}=\tilde{\eta}$ ) such that the image of $\tilde{n}$ on $\Omega_{a}$ is contained in the interior of $Q$. Note that the constants $\tilde{c}_{a b}$ and the weighted curvatures $\Lambda_{a}$ are related through the sense of the relation (8.25), at least for $\Omega_{a}$ bounded.

We will argue by induction on $k$. If $k=3$ we may-modulo a linear coordinate change with translation-assume that $\Omega_{a}$ is a triangle with vertices at $(0,0),(0,1)$, and $(1,0)$. Then an appropriate choice of $\alpha$ and $b$ renders the function

$$
\tilde{\eta}(\mathbf{x})=\alpha \mathbf{x}+\mathbf{b}
$$

consistent with (9.16) and (9.17); such a choice of $\alpha$ and $b$ are unique even if $\alpha$ is regarded as a matrix. (That is, there is a unique affine function satisfying (9.16) and (9.17) if $\Lambda_{a}$ in (9.17) and $\tilde{c}_{a b}$ are given.) As a consequence, $\tilde{\eta}\left(\Omega_{\mathrm{a}}\right)$ is contained in the interior of $Q$.

It remains to establish the existence of $\tilde{\eta}$ for a polygon that has $k+1$ corners assuming the existence of such a field $\tilde{n}$ for any polygon with $p$ corners, where $3 \leq \mathrm{p} \leq \mathrm{k}$. To accomplish this we divide $\Omega_{\mathrm{a}}$ into two polygons $\bar{\Omega}$ and $\bar{\Omega}$ by connecting two nonadjacent corners by a segment $\ell$. Let $\mu$ be a unit normal to $\ell$. If $\operatorname{div} \xi=\Lambda_{a}$ on $\Omega_{a}$, then the normal component $c_{l}=5 \cdot \mu$ of $\boldsymbol{\zeta}$ on $l$ is uniquely determined by the divergence theorem provided $\boldsymbol{\zeta} \cdot \mu$ is assumed to be constant. Let $\bar{Q}$ and $\bar{Q}$ be the polygons constructed from $\bar{\Omega}$ and $\bar{\Omega}$ as in the first paragraph of the proof with $\tilde{c}_{a b}$ replaced by $c_{l}$ on $l$. We may then construct the functions $\bar{\eta}$ and $\bar{\eta}$ for $\bar{\Omega}$ and $\bar{\Omega}$ using the induction assumption. Since the images of $\bar{\eta}$ and $\bar{\eta}$, respectively, are contained in the interiors of $\bar{Q}$ and $\bar{Q}$ the function $\tilde{\eta}$ on $\Omega_{\mathrm{a}}=\bar{\Omega} \cup \bar{\Omega}$ whose restrictions to $\bar{\Omega}$ and $\bar{\Omega}$, respectively, are $\bar{\eta}$ and $\bar{\eta}$, has its image in the interior of the union of $\bar{Q}$ and $\bar{Q}$, which is the interior of $Q$. Further, since $\bar{\eta}$ and $\bar{\eta}$ each satisfy (9.16) and (9.17) with 
5 equal to $\bar{\eta}$ and $\bar{\eta}$ in $\bar{\Omega}$ and $\bar{\Omega}$, respectively, (9.16) is automatically satisfied for $\tilde{n}$. while (9.17) holds for $\tilde{n}$ on $\bar{\Omega}$ and $\bar{\Omega}$. Also, since $\operatorname{tr}(\bar{\eta} \cdot \mu)=\operatorname{tr}(\bar{\eta} \cdot \mu), \operatorname{div} \tilde{\eta}-\Lambda_{a}$ is a function supported on $l$; hence div $\tilde{\eta}-$ $\Lambda_{a}=0$. Thus (9.17) is satisfied for $\tilde{n}$ on all of $\Omega_{a}$, which completes the proof of (a).

(b) Since $\operatorname{tr}\left(\tilde{\eta} \cdot \mu_{a b}\right)=\operatorname{tr}\left(\boldsymbol{b} \cdot \mu_{a b}\right)$ on $\partial \Omega_{a}$, the distributional divergence of $\boldsymbol{r}$ is a function across $\partial \Omega_{\mathrm{a}}$. Thus (9.21) is satisfied.

A consequence of the proof of $(a)$ is

Lemma 9.7. Let $\Omega$ be a polygon in $\mathbb{R}^{2}$ with $l_{b}, b=1,2, \ldots, M$, the edges of $\partial \Omega$, assumed connected. Let $c_{b}, b=1,2, \ldots, M$, and $\wedge$ be constants.

(i) Assume that $\Omega$ is bounded. Then there is a (bounded) piecewise linear function $\eta$ (not necessarily continuous) such that

$$
\begin{array}{ll}
\operatorname{div} \eta=\wedge & \text { in } \Omega, \\
\eta \cdot \mu_{b}=c_{b} & \text { on each edge } l_{b} \text { of } \Omega
\end{array}
$$

if and only if the following compatibility condition is satisfied:

$$
\sum_{b} c_{b} l_{b}=|\Omega| \Lambda
$$

(ii) Assume that $\Omega$ is regularly unbounded and that $\Lambda=0$. Then there is a bounded, piecewise linear function $\eta$ satisfying (9.22).

Proof. Regarding (i), the existence of $\eta$ is established in the proof of Lemma 9.6(a). If $\eta$ satisfying (9.22) exists, then integrating div $\eta$ by parts yields the compatibility condition.

To establish (ii), we note first that if $\Omega$ is all of $\mathbb{R}^{2}$, then any constant $\eta$ provides a solution of (9.22), and if $\Omega$ is a halfspace, say $x_{1} \leq 0$, then $\eta=\left(c_{1}, 0\right)$ provides a solution of (9.22), where $c_{1}$ is the boundary data on the single edge $x_{1}=0$. To complete the proof we have only to consider $\Omega$ unbounded with exactly two unbounded edges $l_{1}$ and $l_{2}$, and with $l_{1}$ and $\ell_{2}$ not parallel. By an affine coordinate change we may restrict attention to the following two cases: 
(L1) $\ell_{1}=\left\{\left(x_{1}, 0\right): x_{1} \geq \sigma\right\}$ for some $\sigma>0$,

$\ell_{2}=\left\{\left(0, x_{2}\right): x_{2} \geq \rho\right\}$ for some $\rho>0$,

$\Omega$ contains $\Omega^{\prime}=\left\{\left(x_{1}, x_{2}\right): x_{1}, x_{2} \geq 0, x_{1} \geq h\right.$ or $\left.x_{2} \geq h\right\}$ for some sufficiently large $h>0$;

(L2) the closure of the complement of a region of (L1)-type.

We will consider only case (L1); (L2) is analogous. We begin by dividing $\Omega$ into four polygonal regions:

$$
\begin{aligned}
& \Omega_{0}=\left\{\left(x_{1}, x_{2}\right) \in \Omega: x_{1} \leq h, x_{2} \leq h\right\} \\
& \Omega_{1}=\left\{\left(x_{1}, x_{2}\right) \in \Omega^{\prime}: x_{1} \geq h, x_{2} \leq h\right\} \\
& \Omega_{2}=\left\{\left(x_{1}, x_{2}\right) \in \Omega^{\prime}: x_{1} \leq h, x_{2} \geq h\right\} \\
& \Omega_{12}=\left\{\left(x_{1}, x_{2}\right) \in \Omega^{\prime}: x_{1} \geq h, x_{2} \geq h\right\} ;
\end{aligned}
$$

then $\Omega_{0}$ is bounded, while $\Omega_{1}, \Omega_{2}$, and $\Omega_{12}$ are unbounded. Next we define a constant $c^{*}$ such that the compatibility condition (9.23) with $\Lambda=0$ is valid for $\Omega_{0}$ with $c_{b}=c^{*}$ on the portion of $\partial \Omega_{0}$ that lies on the line $x_{2}=h$ and on the portion of $\partial \Omega_{0}$ that lies on the line $x_{1}=h$. By part (i) there is a piecewise linear function $\eta$ on $\Omega_{0}$ satisfying (9.22) with $\Lambda=0$. We extend $\eta$ to all of $\Omega$ by defining $\eta=\left(c^{*},-c_{1}\right)$ on $\Omega_{1}, \eta=\left(-c_{2}, c^{*}\right)$ on $\Omega_{2}$, and $\eta=\left(-c_{2},-c_{1}\right)$ on $\Omega_{12}$; the resulting function $\eta$ on $\Omega$ is then bounded, piecewise linear, and consistent with (9.22) (in the sense of distributions).

The existence of $\zeta$ in (9.17) leads to the existence of the CahnHoffman vector $\xi$; that is, a function $\xi$ on $\&$ such that

(C1) $\operatorname{div} P \xi_{\boldsymbol{F}}=-\Lambda_{\mathrm{a}}$ on each facet $F_{\mathrm{a}}$ (cf. (2.4);

(C2) given any edge $l$ of $\&$, the projection of $\xi$ on to the plane perpendicular to $l$ is continuous across $l$;

(C3) $\xi \in \partial \gamma(n)$ on each facet of 8 .

Theorem 9.8. Let \& be a crystalline surface consistent with (W1). Then there is a piecewise linear function $\xi$ on $\&$ that satisfies (C1)-(C3) if and only if (W2) holds.

Proof. We first assume that 8 is bounded and construct $\xi$ on each facet $F_{a}$. Thus choose $F_{a}$ and, without loss in generality, assume that 
$n_{a}=(0,0,1)$. It suffices to solve (9.16) and (9.17) subject to the restriction $(*)$ given after (9.18); indeed, this system has a piecewise linear solution. $\boldsymbol{E}$ as in Lemmas 9.6 and 9.7. Further, we may use the homogeniety of $\gamma$ to conclude that $\xi=(-\boldsymbol{\xi},-\gamma(0,0,1)) \in \partial \gamma\left(n_{a}\right)$. Rotating coordinates yields a solution $\xi$ of (C1) and (C3).

If (W2) holds, then by Lemma 9.3 ,

$$
\boldsymbol{E}_{\mathrm{a}} \cdot \mu_{\mathrm{a}}=-\boldsymbol{\xi} \cdot \mu_{\mathrm{ab}}=-\tilde{c}_{\mathrm{ab}}=-c_{\mathrm{ab}}, \quad \mathrm{n}_{\mathrm{a}}=(0,0,1),
$$

on the $(a, b)$-edge, where $F_{b}$ is a facet adjacent to $F_{a}$. By (3.15) or (3.19), this yields (C2). Finally, if (W2) does not hold, then $-\boldsymbol{\xi} \cdot \mu_{a b}=-\tilde{c}_{a b}$ for some choice of b. Thus (C2) does not hold.

Finally, if $\&$ contains an unbounded facet then the construction in Lemma 9.7 is satisfied for $\boldsymbol{\xi}$, and the remainder of the proof is as above. 
10. COMPARISON PRINCIPLE FOR ADMISSIBLE CRYSTALLINE MOTIONS

We continue to assume, as prescribed:

(i) a set $\Omega$ of admissible orientations;

(ii) for each $n \in \mathcal{N}$, an interfacial free energy $\hat{\gamma}(n)>0$ and a kinetic modulus $\beta(n)>0$;

We now establish the main result of the paper: a comparison principle for admissible crystalline motions \& whose facets $F_{a}$ evolve according to the facet evolution equation

$$
\beta_{a} V_{a}(t)=\Lambda_{a}(t)-U
$$

with $\beta_{a}=\beta\left(n_{a}\right)$ and $\Lambda_{a}(t)$ the energetically-weighted curvature defined in (6.5). Our proof of the comparison principle is based on Theorem 9.1, which characterizes the right side of the evolution equation (9.1) in terms of the canonical subdifferential $\partial^{\circ} \Phi(u)$.

Before stating the comparison principle, we note that if the set $\boldsymbol{\eta}$ is acute, then given any admissible crystalline surface $\&$ and any facet $F_{a}$ of $8, \&$ is essentially the graph of a function $u_{a}$ near $F_{a}$ (cf. Remark (3) of Section 8a). Moreover, modulo a translation and rotation of coordinates, $u_{a}$ may be chosen so that $F_{a}$ is contained in the set $\left\{u_{a}=0\right\}$. Let $\delta_{1}$ and $s_{2}$ be crystalline surfaces. We say that $\delta_{2}$ encloses $\delta_{1}$ if the corresponding closed polyhedra $\Gamma_{1}$ and $\Gamma_{2}\left(8_{1}=\partial \Gamma_{1}, 8_{2}=\partial \Gamma_{2}\right)$ satisfy $\Gamma_{1} \subset \Gamma_{2}$.

Theorem 10.1. Assume that the set $\mathfrak{n}$ of admissible orientations is acute. Let $\delta_{1}$ and $\delta_{2}$ be bounded, strongly admissible crystalline motions with common duration $T$, and assume that the facets of $s_{1}$ and $s_{2}$ evolve according to the facet evolution equation (10.1) with possibly different bulk free-energies $U_{1}$ and $U_{2}$, respectively. Let

$$
\mathrm{U}_{1} \geq \mathrm{U}_{2}
$$

and assume that $\delta_{2}(0)$ encloses $\delta_{1}(0)$. Then $\delta_{2}(t)$ encloses $\delta_{1}(t)$ for $0 \leq t<T$ provided each corner of one of the $\delta_{\mathrm{i}}$ is a triple junction if $\mathrm{U}_{1}=\mathrm{U}_{2}$. 
The proof depends on a. natural extension of a maximum principle for crystals in the plane established in [16]. Let $\delta_{1}=\partial \Gamma_{1}$ and $\delta_{2}=\partial \Gamma_{2}$ be crystalline surfaces. We say that $\delta_{1}$ touches $\delta_{2}$ at $x_{0}$ from inside if $x_{0} \in \delta_{1} \cap \delta_{2}$, and if there is an open neighborhood $W$ of $x_{0}$ such that:

(a) $W$ contains all facets of $\delta_{1}$ that contain $x_{0}$ or all facets of $\&_{2}$ that contain $\mathbf{x}_{0}$; and

(b) $\Gamma_{1} \cap W \subset \Gamma_{2} \cap W$.

There are exactly six possibilities for the touching of $\delta_{1}$ and $\delta_{2}$ at $x_{0}$ :

(1) facet-facet: there are facets $F_{i}$ of $\delta_{i}$ such that $F_{1} \cap F_{2}$ is a nontrivial polygon containing $\mathrm{x}_{0}$;

(2) edge-edge: (a touching that is not facet-facet) for which there are edges $l_{i}$ of $\delta_{i}$ such that $\ell_{1} \cap l_{2}$ is a nontrivial line segment containing $\mathbf{x}_{0}$;

(3) facet-edge: (a touching that is neither facet-facet nor edge-edge) for which there are an edge $l$ of one of the crystals and a facet $F$ of the other such that $\ell \cap F$ is a nontrivial line segment containing $x_{0}$;

(4) facet-corner: (a touching that is not facet-facet, not edge-edge, and not facet-edge) for which there are a corner $c$ of one of the crystals and a facet $F$ of the other such that $\{c\} \cap F$ consists of $x_{0}$ lying in the interior of $F$;

(5) edge-corner: (a touching that is not facet-facet, not edge-edge, not facet-edge, and not facet-corner) for which there are a corner $c$ of one of the crystals and an edge $l$ of the other such that $\{c\} \cap l$ consists of $x_{0}$ with $x_{0}$ not an endpoint of $l$;

(6) corner-corner: (a touching that is not facet-facet, not edge-edge, not facet-edge, not facet-corner, and not edge-corner) for which $\mathbf{x}_{0}$ is a corner of both crystals.

(The terminology here is somewhat different from that of [16]; in particular, the improper edge-edge touching in [16] is included in (1) above.)

Lemma 10.1. Let $\delta_{1}$ and $\delta_{2}$ be admissible crystalline surfaces such that $\delta_{1}$ touches $\delta_{2}$ at $\mathbf{x}_{0}$ from inside.

(i) Then the touching cannot be facet-edge. It can be edge-edge only if the set of orientations of facets of $\delta_{1}$ meeting at $\mathrm{x}_{0}$ equals the corresponding set for $B_{2}$. 
(ii) Let $\delta_{1}$ and $\delta_{2}$ be strongly admissible. Then the touching cannot be facet-corner nor can it be edge-corner. It can be corner-corner only if the set of orientations of facets of $\delta_{1}$ meeting at $\mathrm{x}_{0}$ equals the corresponding set for $\&_{2}$.

Proof (Lemma). The proof of (i) is identical to the proof of (i) of the Maximum Principle of [16]. To verify (ii), let $x_{0}$ be a corner of, say, $\&_{1}$, and let $\eta_{0}$ be the set of orientations of facets meeting at $x_{0}$. Suppose that $s_{1}$ touches $\delta_{2}$ at $x_{0}$ from inside. By Proposition 5.1, $\eta_{0}$ is complete. Let $F$ be a facet of $\delta_{2}$ that contains $x_{0}$. Since $\eta_{0}$ is complete, the orientation $n$ of $F$ is contained in $\eta_{0}$, and therefore facet-corner and edge-corner touchings are not possible for $F$. In addition, the set of orientations of facets of $\delta_{2}$ meeting at $x_{0}$ equals $\pi_{0}$.

The next result is an analog of the classical maximum principle.

Theorem 10.2. Let $\delta_{1}$ and $\delta_{2}$ be bounded, strongly admissible crystalline surfaces such that $\delta_{1}$ touches $\&_{2}$ at $\mathbf{x}_{0}$ from inside. Then:

(i) There are facets $F_{1}$ and $F_{2}$ of $\delta_{1}$ and $\delta_{2}$ such that $x_{0} \in F_{1} \cap F_{2}$ and such that the orientation of $F_{1}$ equals that of $F_{2}$. Further, the touching is either facet-facet, edge-edge, or corner-corner; and there is a point $y_{0}$ sufficiently close to (possibly equal to) $\mathbf{x}_{0}$ such that $8_{1}$ touches $\delta_{2}$ at $y_{0}$ from inside and such that the set of orientations of facets of $\&_{1}$ meeting at $y_{0}$ equals the corresponding set for $\delta_{2}$.

(ii) For $\mathfrak{N}$ acute, if $F_{1}$ and $F_{2}$ are facets of $\delta_{1}$ and $\delta_{2}$ with $x_{0} \in F_{1} \cap F_{2}$ and with the orientation of $F_{1}$ equal to that of $F_{2}$, then the energetically weighted curvatures $\Lambda_{1}$ and $\Lambda_{2}$ of $F_{1}$ and $F_{2}$ satisfy

$$
\Lambda_{1} \leq \Lambda_{2}
$$

Strong admissibility is needed only for the cases in which $F_{1}$ and $F_{2}$ meet at edges or at corners. Further, (W3) is not needed if the touching is facet-facet.

Assertion (i) of Theorem 10.2 is a direct consequence of Lemma 10.2. A 
key element of the proof of assertion (ii) is a maximum (comparison) principle for subdifferentials. Let $\Phi_{\psi}$ denote the functional on $H=L^{2}(\Omega)$ defined by (9.5).

Lemma 10.2. Let $u_{1}$ and $u_{2}$ be Lipschitz continuous functions on $\Omega$ with $u_{1} \leq u_{2}$ in $\Omega$. Let $\psi_{i}$ be the trace of $u_{i}$ on $\partial \Omega \quad(i=1,2)$. Let $u_{1}=u_{2}$ in an open set $\Omega^{\prime} \subset \Omega$. Assume that $\partial^{\circ} \Phi_{i}\left(u_{i}\right)(x)$ is continuous for $\mathbf{x}$ in $\Omega^{\prime}$, where $\Phi_{\mathrm{i}}=\Phi_{\psi_{\mathrm{i}}}$. Then

$$
-\partial^{\circ} \Phi_{1}\left(u_{1}\right)(x) \leq-\partial^{\circ} \Phi_{2}\left(u_{2}\right)(x) \quad \text { for all } x \in \Omega^{\prime} \text {. }
$$

Proof (Lemma).

$1^{\circ}$ We consider the initial-value problem for an abstract differential equation in $\mathrm{H}$ :

$$
\begin{aligned}
& d U_{i} / d t \epsilon-\partial \Phi_{i}\left(U_{i}\right) \text { for a. e. } t>0, \\
& \left.U_{i}\right|_{t=0}=u_{i} .
\end{aligned}
$$

Since $\Phi_{i}$ is lower semicontinuous and convex (Lemma 9.1), the abstract theory yields the unique existence of a solution $U_{i} \in C([0, \infty), H)$ with $U_{i}$ absolutely continuous in $[0, \infty)$ (cf., e.g., [5, IV, Theorem 2.1]).

$2^{\circ}$ Since $u_{1} \leq u_{2}$, it follows that $U_{1}(t, x) \leq U_{2}(t, x)$ for a.e. $x$ for all $t \geq 0$. This may be verified by approximating $j$ in (9.5) by smooth, convex, coercive $j_{\varepsilon}$ as in the proof of [13. Theorem 3.3]. Here we shall only sketch the proof. Since the comparison principle holds for approximate solutions $U_{\varepsilon i}, U_{\varepsilon 1} \leq U_{\varepsilon 2}$ in $[0, \infty) \times \Omega$. It is possible to take approximate $j_{\varepsilon}$ so that the stability theorem [30, Remark 2.7] applies; hence $U_{\varepsilon i} \rightarrow U_{i}$ in $C([0, T], H)$ for each $T>0$. This yields $U_{1}(t, \cdot) \leq U_{2}(t, \cdot)$ a.e. in $\Omega$ for all $t \geq 0$.

$3^{\circ}$ The generator of the evolution equation in $1^{\circ}$ is given by $-\partial^{\circ} \Phi_{i}$ (cf. [5]). Thus 


$$
\begin{aligned}
-\partial^{\circ} \Phi_{1}\left(u_{1}\right) & =\lim _{t \rightarrow 0^{+}} t^{-1}\left[U_{1}(t, \cdot)-u_{1}\right] \\
& =\lim _{t \rightarrow 0^{+}} t^{-1}\left[U_{2}(t, \cdot)-u_{1}\right] \\
& =\lim _{t \rightarrow 0^{+}} t^{-1}\left[U_{2}(t, \cdot)-u_{2}\right]=-\partial^{\circ} \Phi_{2}\left(u_{2}\right) \quad \text { a.e. in } \Omega^{\prime},
\end{aligned}
$$

where "lim" denotes the $L^{2}$ strong limit on $\Omega^{\prime}$. Since $\partial^{\circ} \Phi_{i}\left(u_{i}\right)$ is continuous in $\Omega$ ', this completes the proof.

Proof (Theorem 10.2). Without loss in generality we may assume that $s_{i}$ is the graph of a function $u_{i}$ near $u_{i}$ such that $u_{i}=0$ on $P\left(F_{i}\right)=\Omega_{i}$ and that the outward unit normal to the crystalline surfaces on the facets $F_{i}$ point upward.

Case $A$. The intersection of $F_{1}$ and $F_{2}$ is a nontrivial polygon $F$.

$1^{\circ}$ Suppose that, for each $i, \delta_{i}$ is a graph of $u_{i}$ in a common open set $\Omega$ containing

$$
R_{0}=\Omega_{1} \cup \Omega_{2} \text {. }
$$

with $\Omega$ consistent with (G1)-(G3). Since $\delta_{1}$ touches $\delta_{2}$ from inside and since the intersection of $\delta_{1}$ and $S_{2}$ contains $F, u_{1} \leq u_{2}$ in $\Omega$ with $u_{1}=u_{2}$ on $F$. Thus, by Lemma $10.2,-\partial^{\circ} \Phi_{1}\left(u_{1}\right)(x) \leq-\partial^{\circ} \Phi_{2}\left(u_{2}\right)(x)$ for all $x \in P(F) \subset R_{0}$, where $\Phi_{i}=\Phi$ is the functional defined in (9.5) with $\psi=\operatorname{tr} u_{i}$ on $\partial \Omega$ and $U=0$.. Thus, since $\Lambda_{i}$ is given by $-\partial^{\circ} \Phi_{i}\left(u_{i}\right)(x)$ (cf. Theorem 9.1), this yields $\Lambda_{1} \leq \Lambda_{2}$.

$2^{\circ}$ We now complete the proof of Case $A$ by showing that $\Lambda_{1} \leq \Lambda_{2}$ without assuming that $\delta_{i}$ is a graph of $u_{i}$ in a common open set $\Omega$ containing $R_{0}$. Since $\delta_{i}$ is the graph of a function $u_{i}$ near $F_{i}(i=1,2)$, there is a polygon $\Omega_{i}^{\prime}$ containing $\Omega_{i}$ such that (G1) holds for $\Omega_{i}^{\prime}$ i.e.,

$$
\delta_{i} \cap \Omega_{i}^{\prime} \times \mathbb{R}=\left\{\left(x, u_{i}(x)\right): x \in \Omega_{i}^{\prime}\right\}
$$

and such that $\Omega_{i}^{\prime}$ satisfies (G2)-(G3) in section $8(a)$ for $\Omega$. Let $\bar{F}_{1}$ be any facet adjacent to $F_{1}$ such that $u_{1} \leq 0$ on $P\left(\bar{F}_{1}\right) \cap \Omega^{\prime}{ }_{1}$ and such that $P\left(\bar{F}_{1}\right) \cap \Omega^{\prime}{ }_{1}$ intersects the interior of $\Omega_{2}$. 
For $\delta>0$ we set

$$
v_{1}(x)=\max \left\{u_{1}(x),-\delta\right\} \text { for } x \in P\left(\bar{F}_{1}\right) \cap \Omega^{\prime}{ }_{1}
$$

and

$$
v_{1}(x)=-\delta \text { for } x \in \Omega_{2}^{\prime} \backslash \Omega_{1}^{\prime}
$$

Similarly, let $\bar{F}_{2}$ be any facet adjacent to $F_{2}$ such that $u_{2} \geq 0$ on $P\left(\bar{F}_{2}\right) \cap \Omega_{2}^{\prime}$ and such that $P\left(\bar{F}_{2}\right) \cap \Omega_{2}^{\prime}$ intersects the interior of $\Omega_{1}$.

For $\delta>0$ we set

$$
v_{2}(x)=\min \left\{u_{2}(x), \delta\right\} \text { for } x \in P\left(\bar{F}_{2}\right) \cap \Omega^{\prime}{ }_{2}
$$

and

$$
v_{2}(x)=\delta \text { for } x \in \Omega^{\prime}{ }_{1} \backslash \Omega^{\prime}{ }_{2}
$$

For sufficiently small $\delta$ and for some $R$ the graph of $v_{i}$ on $R$ is a surface, which we denote by $S_{i}(i=1,2)$. Since $\delta_{2}$ encloses $\delta_{1}, S_{2}$ lies above $S_{1}$ near $R_{0}$; and $s_{2}$ \{resp., $\delta_{1}$ \} is located above \{resp., below\} both $S_{2}$ and $S_{1}$ near $\Omega_{2}$ \{resp., $\Omega_{1}$ \} for sufficiently small $\delta$. Further, we may assume that $S_{1}$ and $S_{2}$ satisfy (W1) and (W2) over $R$ if $S_{1}$ and $\delta_{2}$ satisfy (W1) and (W2). (Of course, if $\delta_{1}$ and $\delta_{2}$ satisfy (W3), then so do $S_{1}$ and $S_{2}$ for sufficiently small $\delta$, but (W3) is not needed for this step.) We may also assume that $R$ satisfies (G1)-(G3) for $S_{1}$ and $S_{2}$. In a neighborhood of $\Omega_{1}$ \{resp:, $\Omega_{2}$ \}: $\delta_{1}$ \{resp., $S_{2}$ \} is the graph of $u_{1}$ \{resp., $\left.u_{2}\right\} ; S_{1}$ \{resp., $\left.s_{2}\right\}$ is the graph of $v_{1}$ \{resp., $\left.v_{2}\right\} ; u_{1} \leq v_{1}$ \{resp., $\left.v_{2} \leq u_{2}\right\}$. Thus Lemma 10.2 yields

$$
\begin{array}{ll}
-\partial^{\circ} \Phi_{1}\left(u_{1}\right)(x) \leq-\partial^{\circ} \Phi_{1 n}\left(v_{1}\right)(x), & x \in \Omega_{1}, \\
-\partial^{\circ} \Phi_{2 *}\left(v_{2}\right)(x) \leq-\partial^{\circ} \Phi_{2}\left(u_{2}\right)(x), & x \in \Omega_{2},
\end{array}
$$

where $\Phi_{i n}(i=1,2)$ is defined by (9.5) with $\Omega=\Omega_{i}, U=0$, and $\psi=\operatorname{tr} v_{i}$ on $\partial \Omega_{\mathrm{i}}$, while $\Phi_{\mathrm{i}}$ is defined similarly with $\psi=\operatorname{tr} u_{\mathrm{i}}$ on $\partial \Omega_{\mathrm{i}}$. Next, comparing $v_{1}$ and $v_{2}$ on $R$ using Lemma 10.2,

$$
-\partial^{\circ} \Phi_{1 * *}\left(v_{1}\right)(x) \leq-\partial^{\circ} \Phi_{2 * *}\left(v_{2}\right)(x), \quad x \in R_{0}
$$

where $\Phi_{i * *}$ is defined by (9.5) with $\Omega=R, U=0$, and $\psi=\operatorname{tr} v_{i}$ on $\partial R$. 
Fortunately, Theorem 9.1 implies that

$$
-\partial^{\circ} \Phi_{i *}\left(v_{i}\right)(x)=-\partial^{\circ} \Phi_{i * *}\left(v_{i}\right)(x), \quad x \in \Omega_{i},
$$

and since the facet $v_{i}=0$ is $F_{i}$, independently of $R$,

$$
-\partial^{\circ} \Phi_{1}\left(u_{1}\right)(x) \leq-\partial^{\circ} \Phi_{2}\left(u_{2}\right)(x), \quad x \in R_{0}
$$

thus Theorem 9.1 yields $\Lambda_{1} \leq \Lambda_{2}$.

(Note that Step $2^{\circ}$ actually includes Step $1^{\circ}$; we give Step $1^{\circ}$ explicitly as it contains the central idea of the proof.)

Case $B$. The intersection of $F_{1}$ and $F_{2}$ is along an edge of each or at a corner of each.

Since $\delta_{1}$ touches $\delta_{2}$ at $x_{0}$ from inside, there is a neighborhood $W$ of $x_{0}$ such that $\Gamma_{1} \cap W \subset \Gamma_{2} \cap W$ and $W$ contains all facets of $\delta_{1}$ that contain $\mathbf{x}_{0}$ or all facets of $\delta_{2}$ that contain $\mathbf{x}_{0}$. Assume, without loss in generality, that $W$ contains all facets of $s_{1}$ that contain $x_{0}$. We use a sliding argument. Choose a facet $J$ of $s_{1}$ that is different from $F_{1}$ and that contains $x_{0}$. Let $J_{b}, b=1,2, \ldots, M$, denote the facets adjacent to $J$ and let $l_{c}, c=1,2, \ldots, m$, denote those edges of the facets $J_{b}$ whose intersections with $J$ are corners. Let $\bar{l}_{c}$ denote the line containing $\ell_{c}$, $c=1,2, \ldots, m$. We move $J$ a small amount $\delta$ in the direction of its orientation letting $\mathrm{J}$ grow so that its corners remain on $\bar{l}_{c}$. We denote the new facet by $J_{\delta}$. For $\delta$ sufficiently small this procedure defines a new crystalline surface $S_{\delta}$ that satisfies (W1)-(W3) if $\delta_{1}$ does. (We now invoke (W3), because without it $S_{\delta}$ may not satisfy (W2) even when $\&_{1}$ satisfies (W1) and (W2).) At the points of $J$ that intersect $\&_{2}$ we have the orientational relations asserted in Lemma 10.1; hence, for $\delta$ sufficiently small, $S_{\delta}$ touches $\delta_{2}$ at $x_{0}$ from inside. On the other hand, by the construction, $\&_{1}$ touches $S_{\delta}$ at $x_{0}$ from inside, and both touchings are facet-facet; that is, there is a facet $F_{8}$ of $S_{8}$ such that $F_{8} \cap F_{1}$ and $F_{8} \cap F_{2}$ are nontrivial polygons. Let $\Lambda_{\delta}$ denote the energetically weighted curvature of of $F_{8}$. By Case $A, \Lambda_{1} \leq \Lambda_{8}$ and $\Lambda_{8} \leq \Lambda_{2}$; so that $\Lambda_{1} \leq \Lambda_{2}$.

Remark. In the sliding argument the new crystal $S_{6}$ has only triple junctions at corners in $W$ for all sufficiently small $\delta$. In some sense this 
indicates the pathological nature of nontriple junctions, as they are unstable under small perturbations.

Proof (Theorem 10.1). Conceptually, the proof is similar to that of the First Comparison Theorem of [16].

$1^{\circ}$ The case $U_{1}>U_{2}$. Assume that $\delta_{2}(t)$ does not enclose $\delta_{1}(t)$ at some $t \in(0, T)$. Then

$$
0 \leq t_{0}=\sup \left\{t: \quad \delta_{2}(t) \text { encloses } \delta_{1}(t)\right\}
$$

satisfies $t_{0}<T$. By continuity and Lemma 10.1, $\delta_{2}\left(t_{0}\right)$ encloses $\delta_{1}\left(t_{0}\right)$ and $s_{1}\left(t_{0}\right)$ touches $s_{2}\left(t_{0}\right)$ at some point $x_{0}$ from inside with the touching of one of the following three types:

$(\alpha)$ facet-facet. There are facets $F_{i}$ of $\delta_{i}\left(t_{0}\right)$ such that $F_{1} \cap F_{2}$ is a nontrivial polygon containing $\mathbf{x}_{0}$. At $t=t_{0}$ the normal velocities $V_{i}$ of $F_{i}$ in the direction of their common orientation satisfy $V_{1} \geq V_{2}$.

( $\beta$ ) edge-edge. There are edges $\ell_{i}$ of $\delta_{i}\left(t_{0}\right)$ such that $\ell_{1} \cap l_{2}$ is a nontrivial line segment containing $\mathbf{x}_{0}$. Moreover, $l_{i}$ is an edge of adjacent facets $F_{i 1}$ and $F_{i 2}$ of $\delta_{i}\left(t_{0}\right),(i=1,2)$ and, by Lemma $10.2, F_{1 j}$ and $F_{2 j}$ have the same orientation $n_{j}(j=1,2)$ (by renumbering if necessary). Further, at $t=t_{0}$ the normal velocities $V_{1 j}$ and $V_{2 j}$ of $F_{1 j}$ and $F_{2 j}$ in the direction of $n_{j}$ satisfy $V_{11} \geq V_{21}$ or $V_{12} \geq V_{22}$.

( $\gamma$ ) corner-corner: By Lemma 10.1 the sets $\left\{F_{i j}, j=1,2, \ldots, M\right\} \quad(i=1,2)$ of facets of $\delta_{i}\left(t_{0}\right) \quad(i=1,2)$ meeting at the corner $x_{0}$ have the following properties for $j=1,2, \ldots, M$ :

(i) $F_{1 j}$ and $F_{2 j}$ have the same orientation $n_{j}$;

(ii) $F_{1 j} \cap F_{2 j}=\left\{x_{0}\right\}$.

Further, at $t=t_{0}$ the velocities $V_{1 j}$ and $V_{2 j}$ of $F_{1 j}$ and $F_{2 j}$ in the direction $n_{j}$ satisfy $V_{1 j} \geq V_{2 j}, j=1,2, \ldots, M$.

By Theorem 10.2 we have, for the cases $(\alpha)-(\gamma)$, respectively:

( $\alpha) \quad \Lambda_{1} \leq \Lambda_{2}$ with $\Lambda_{i}$ the (energetically weighted) curvature of $F_{i}$.

(B) $\Lambda_{1 j} \leq \Lambda_{2 j}(j=1,2)$ with $\Lambda_{i j}$ the curvature of $F_{i j}$.

(r) $\Lambda_{1 j} \leq \Lambda_{2 j}(j=1,2, \ldots, M)$ with $\Lambda_{i j}$ the curvature of $F_{i j}$.

Using the facet equation (10.1) in each of the cases $(\alpha)-(\gamma)$ we see that the 
signs of the velocities and curvatures yjeld in each case the inequality $\mathrm{U}_{1} \leq \mathrm{U}_{2}$, a contradiction.

$2^{\circ}$ The case $U_{1}=U_{2}$. Assume that each corner of $\&_{2}$ is a triple junction. By a standard theorem on continuous dependence on data, applied to the ODE system (6.10), one can show that for each $\varepsilon>0$ there is a strongly admissible crystalline evolution $\delta_{\varepsilon}$ that satisfies $\delta_{\varepsilon}(0)=\delta_{2}(0)$, that evolves according to the facet equations (10.1) with $U_{2}$ replaced by $\mathrm{U}_{2}-\varepsilon$, and is such that

(i) its duration $T_{\varepsilon}$ satisfies $T_{\varepsilon} \rightarrow T$ as $\varepsilon \rightarrow 0$;

(ii) $\operatorname{dist}\left(\delta_{\varepsilon}(t), \delta_{2}(t)\right) \rightarrow 0$ as $\varepsilon \rightarrow 0$ uniformly for $t$ in any closed interval of $[0, T)$.

Here $\operatorname{dist}\left(\delta_{\varepsilon}(t), \delta_{2}(t)\right)=\inf \left\{|\mathbf{x}-\overline{\mathbf{x}}|: \mathbf{x} \varepsilon \delta_{\varepsilon}(t), \overline{\mathbf{x}} \varepsilon \delta_{2}(t)\right\}$. By Step $1^{\circ}, \delta_{\varepsilon}(t)$ encloses $\delta_{1}(t)$ for $0 \leq t<T_{\varepsilon}$. Letting $\varepsilon \rightarrow 0$ we conclude that $\delta_{2}(t)$ encloses $\delta_{1}(t)$ for $0 \leq t<T$.

If each corner of $\delta_{1}$ (rather than $\delta_{2}$ ) is a triple junction we approximate $\delta_{1}$ instead of $\delta_{2}$ by $\delta_{\varepsilon}$ with $U_{1}$ replaced by $U_{1}+\varepsilon$.

Remarks.

(1) The triple junction property is used only to obtain the approximation $8_{\varepsilon}$.

(2) As in the first comparison theorem in [16], one can prove that $\operatorname{dist}\left(\delta_{\varepsilon}(t), B_{2}(t)\right)$ is nonincreasing in $t$.

(3) As remarked in [16, Section 4], if $U_{1}>U_{2}$, then $S_{2}(t)$ does not touch $s_{1}(t)$ for $0 \leq t<T$.

(4) The second comparison theorem and the comparison theorem for weakly admissible evolving crystals in [16, Section 4] extend to the threedimensional theory discussed here with essentially the same proofs.

The proofs of Remarks (1) and (2) parallel those of the analogous assertions in [16].

Acknowledgment. The work of Giga was supported by the Suhara Memorial Foundation and the Yamada Science Foundation, as well as by a Grant-in Aid for Scientific Research (07044094), International Science Research Program, Joint Research, the Ministry of Education, Science, and 
Culture. The work of Gurtin and Matias was supported by the U. S. Army Research Office and the U. S. National Science Foundation. Much of this research was carried out during visits of Giga and Matias to Carnegie-Mellon University and during a visit of Gurtin to Hokkaido University. 


\section{REFERENCES}

[1] H. Attouch and A. Damlamian, Application des methods de convexite' et monotonie a l'etude de certaines equations quasi lineares, Proc. Roy. Soc. Edinburgh 79A, 107-129 (1977)

[2] S. Angenent and M. E. Gurtin, Multiphase thermomechanics with interfacial structure. 2. Evolution of an isothermal interface, Arch. Rational Mech. Anal. 108, 323-391 (1989)

[3] F. Almgren and J. Taylor, Flat flow is motion by crystalline curvature for curves with crystalline energies, preprint University of Minnesota, Geometry Center

[4] F. Almgren, , J. Taylor and L. Wang, Curvature-driven flows: a variational approach, SIAM J. Control Optim.31, 387-437 (1993)

15] V. Barbu, Nonlinear Semigroups and Differential Equations in Banach Space, Noordhoff, Groningen (1976)

[6] H. Brezis, Monotonicity methods in Hilbert spaces and some applications to nonlinear differential equations, Contributions to Nonlinear Functional Analysis (ed. E. Zarantonello), 101-156 Academic Press, New York (1971)

[7] K. A. Brakke, The motion of a Surface by its Mean Curvature, Princeton University Press (1978)

[8] Y.-G. Chen, Y. Giga, and S. Goto, Uniqueness and Existence of viscosity solutions of generalized mean curvature flow equations, $J$. Diff. Geom. 33, 749-786 (1991)

19] J. W. Cahn and D. W. Hoffman, A vector thermodynamics for anisotropic surfaces - 2. curved and faceted surfaces, Act. Metall. 22, 1205-1214 (1974)

[10] J. W. Cahn and J. Taylor, A contribution to the theory of surface energy minimizing shapes, Scripta Metall. 18, 1117-1120 (1984)

[11] C. M. Elliot, A. R. Gardiner and R. Schatzle, Crystalline curvature flow of a graph in a variational setting, Univ. of Sussex at Brighton, Research Report N 95/06

[12] L. C. Evans and J. Spruck, Motion of level sets by mean curvature, I, J. Differential Geometry 33, 635-681 (1991)

[13] T. Fukui and Y. Giga, Motion of a graph by nonsmooth weighted curvature, Proceedings of the First World Congress of Nonlinear 
Analysts 1 (ed. V. Lakshmikantham), Walter de Gruyter, Hawthorne, Berlin, 47-56 (1995)

[14] M.-H. Giga and Y. Giga, Geometric evolution by nonsmooth interfacial energy, Banach Center Publication, Forthcoming

[15] M.-H. Giga and Y. Giga, Consistency in evolutions by-crystalline curvature, Proceedings of Free Boundary Problems 95 (ed. M. Nie'zgo'dka) (Zakopane, Poland 1995), Forthcoming

[16] Y. Giga and M. E. Gurtin, A comparison theorem for crystalline evolution in the plane, Q. Appl. Math. Forthcoming

[17] P. M. Girão, Convergence of a crystalline algorithm for the motion of a simple closed convex curve by weighted curvature, SIAM J. Numer. Anal. 32, 1443-1474 (1995)

[18] P. M. Girão and R. V. Kohn, Convergence of a crystalline algorithm for the heat equation in one dimension and for the motion of a graph by weighted curvature, Num. Math. 67, 41-70 (1994)

[19] P. M. Girão and R. V. Kohn, The crystalline algorithm for computing motion by curvature, Variational Methods for Discontinuous Structure (eds. R. Serapioni and F. Tomarelli) Birkhauser, Forthcoming

[20] M. E. Gurtin, Thermomechanics of Evolving Phase Boundaries in the Plane, Oxford University Press (1993)

[21] M. E. Gurtin and A. Struthers, Multiphase thermomechanics with interfacial structure. 3. Evolving phase boundaries in the presence of bulk deformation, Arch. Rational Mech. Anal. 112, 97-160 (1990)

[22] D. W. Hoffman and J. W. Cahn, A vector thermodynamics for anisotropic surfaces - 1. Fundamentals and applications to plane surface junctions, Surface Sci. 31, 368-388 (1972)

[23] H. M. Soner, Motion of a set by the curvature of its boundary, J. Differential Equations 101, 313-372 (1993)

124] A. Stancu, Uniqueness of self-similar solutions for a crystalline flow, preprint

[25] J. E. Taylor, Constructions and conjectures in crystalline nondifferential geometry, Monographs in Pure and Applied Mathematics 55, 321-336 (ed. B. Lawson and K. Tanenblat) Pitman, London (1991)

[26] J. E. Taylor, Mean curvature and weighted mean curvature, Act. 
Metall. 40, 1475-1485 (1992)

[27] R. Teman, Navier-Stokes Equations, North Holland, Amsterdam (1977)

[28] J. E. Taylor and J. W. Cahn, Catalog of saddle shaped surfaces in crystals, Act. Metall. 34, 1-12 (1986)

[29] J. E. Taylor, J. W. Cahn, and C. Handwerker, Geometric models of crystal growth, Act. Metall. 40, 1443-1474 (1992)

[30] J. Watanabe; Approximation of nonlinear problems of a certain type, Lec. Notes Num. Appl. 1, 147-163 North Holland, Amsterdam (1979) 